\title{
ON THE STABILITY OF RELATIVISTIC SYSTEMS
}

JOHN L. FRIEDMAN AND BERNARD F. SCHUTZ

Reprinted for private circulation from

The Astrophystcal Jourmal, Vol. 200, No. 1, Part 1, 1975 August 15 - 1975. The American Astronomical Society. All rights reserved.

pemroxo in o.s. A. 
The Astrophysical Journal, 200: 204-220, 1975 August 15

C 1975. The American Astronomical Society. Alt rights reserved. Printed in U.S.A.

\title{
ON THE STABILITY OF RELATIVISTIC SYSTEMS*
}

John L. Friedman and Bernard F. Schutz

Department of Physics, Yale University, New Haven, Connecticut

Received 1975 January 13

\begin{abstract}
Using methods applicable to all classical radiative field theories, a necessary and sufficient condition is derived for the onset of instability along a sequence of stationary equilibria in general relativity. A related result is that instability in any mode can set in oniy when its frequency vanishes. Astrophysical implications are briefly discussed.
\end{abstract}

Subject headings: hydrodynamics — instabilities - relativity

\section{INTRODUCTION}

In this paper we discuss the stability of stationary configurations in general relativity, deriving an exact criterion for the first appearance of an unstable linear perturbation along a sequence of equilibria that do not contain event horizons. Our main concern is with perfect fluids; but the criterion applies to a wide range of sources of the metric whose equations can be derived from a Lagrangian, and we append an extension of the formalism to electromagnetism and elastic solids. The criterion for fluids sharply constrains the amount of rotation permitted in compact stars and probably eliminates very high-mass white dwarfs as models for binary X-ray sources.

The criterion's principal virtue is to elucidate in a simple way the role that gravitational radiation plays, confirming a previous conjecture (Chandrasekhar and Friedman 1973) that instabilities set in only through zerofrequency modes, and justifying a previous criterion (Schutz 1972) that at the time was not believed exact in locating points of marginal stability. It is gauge-independent (apart from asymptotic regularity conditions at infinity) and involves no special choice of hypersurface. A drawback is its assumption that the normal modes vary continuously along the sequence of equilibrium models.

In 1967, Lynden-Bell and Ostriker completed an extension to differentially rotating Newtonian configurations of a variational method, using Lagrangian perturbations, that had been developed in earlier work by Chandrasekhar (1964), Clement (1964, 1965), and Lebovitz (1961). Subsequently, Chandrasekhar and Friedman (1972a, b) derived analogous variational expressions for perturbations of uniformly rotating stars in general relativity, which have recently been extended to the case of differential rotation by Will (1973). At the same time Taub (1969) developed a covariant second variational formalism for treating fluid perturbations, which one of us (Schutz 1972) modified and used to derive an energy condition that was sufficient for stability and which is here proved to be necessary as well. To a certain extent this paper simply applies Taub's formalism to the case of harmonic time dependence, and thereby translates the Lynden-Bell and Ostriker paper into general relativity. Because of the increasing generality of the historical development, nearly all previous expressions for the characteristic frequencies of oscillation that appear in the papers mentioned above are special cases of equation (3.21) below.

We find that gravitational radiation has the same effect in the exact theory and for arbitrary equation of state and rotation law that Chandrasekhar (1970) found in his radiation-reaction analysis of the uniformly rotating, uniform-density Maclaurin spheroid; there, for sufficiently rapid rotation, instability to the stationary Dedekind ellipsoid sets in through a nonaxisymmetric deformation. Prior to that point, radiation damps the oscillation; but beyond it (for greater values of the angular velocity), the radiation reaction term effectively drives the instability, by allowing a transition to the lower energy nonaxisymmetric state that conservation of angular momentum would forbid in the absence of dissipation.

From this result and work by Ipser and Detweiler (1973) and Ipser (1975) showing that dynamical instability of nonrotating stars is always associated with radial pulsations, it appears that (dynamical) instabilities in general relativity occur in only two ways: through axisymmetric pulsation when the star's adiabatic index is too small or its density too large; and through nonaxisymmetric perturbations when the rotation is too rapid. In both cases the marginally stable mode is quasi-stationary; and in each instance, relativistic effects cause the instability to set in sooner than in Newtonian theory, with sharp differences possible even in stars whose equilibrium structure is nearly Newtonian.

Our methods are actually applicable not only to general relativity but to any field theory with radiation. Therefore in $\$ 11$ we derive some basic results for Lagrangian theories and their second variations. From this, in the case of harmonic time dependence, a standard equation for the characteristic frequencies emerges, from which follow

- Work supported by the National Science Foundation under grant GP-36317. 
some restrictions on unstable modes. In $\S$ III, a Lagrangian perturbation formalism due to Taub (1969) and to Carter $(1973 a)$ is presented and used to derive the corresponding frequency equation in general relativity appropriate to perturbations of stationary (but not necessarily axisymmetric) space-times containing perfect fluid. In $\S I V$, we obtain our main results, viz., (1) a proof that any mode of oscillation can become unstable along a smooth sequence of equilibrium configurations only when its eigenfrequency vanishes, and (2) a minimum principle for the onset of instability involving a functional whose value is independent of gauge (apart from asymptotic regularity conditions) and of the null or spacelike hypersurface on which it is evaluated. Finally, in $\S \mathrm{V}$, we speculate briefly on possible astrophysical implications of the work. The Newtonian limit of the criterion and the modifications necessary to extend it to electromagnetic fields and elastic solids are presented in appendixes.

Our notational conventions in the sections on relativity are: signature +2 , Latin indices from 0 to 3 , Greek from 1 to 3 .

\section{STABILITY AND THE SECOND VARIATION FOR LAGRANGIAN SYSTEMS}

Because our theorems rest largely on features common to a wide class of relativistic systems, it is useful to begin by establishing some basic results in a general Lagrangian theory (restricted in this section to flat space for simplicity). Whenever the equations governing a system are derivable as the Euler-Lagrange equations of some Lagrangian, the linearized equations for the first-order difference between two nearby solutions are also derivable from a Lagrangian (the "second variation") whose form is easily written down. Closely related is the fact, proved below, that the linearized equations are a symmetric differential system on functions that vanish sufficiently fast at the boundary of a (possibly infinite) region. This leads to a weak stability property common to all Lagrangian systems.

Thus suppose that $y_{A}(A=1, \ldots, N)$ are $N$ fields on a region $\Omega$ of flat space and that $\&\left(x^{i}, y_{A}, y_{A, i}, \ldots\right)$ is a Lagrangian density, real in the sense that $\mathcal{L}\left(x^{i}, y_{A}{ }^{*}, y_{A}{ }^{*}, \ldots\right)=\mathcal{L}^{*}\left(x^{i}, y_{A}, \ldots\right)$, with corresponding action $I=\int_{\Omega} \Omega$. Requiring $\delta I=0$ for perturbations vanishing sufficiently rapidly at the boundary $\partial \Omega$ leads to the field equations

$$
0=F^{A}(y)=\frac{\partial \mathfrak{I}}{\partial y_{A}}-\partial_{i} \frac{\partial \mathfrak{I}}{\partial y_{A, i}}+\partial_{i} \partial_{j} \frac{\partial \mathfrak{I}}{\partial y_{A, i j}}-\cdots
$$

Let $y_{A}$ and $y_{A}+\delta y_{A}$ be two nearby solutions to these equations. We define the corresponding Eulerian perturbation in a quantity $Q\left(x^{i}, y_{A}, y_{A, i}, \ldots\right)$ by

$$
\delta Q=\delta y_{A} \frac{\partial Q}{\partial y_{A}}+\delta y_{A, i} \frac{\partial Q}{\partial y_{A, i}}+\cdots
$$

Then the perturbed field $\delta y_{A}$ satisfies to first order the linearized field equations

$$
0=\delta F^{A}=\delta\left[\frac{\partial \mathcal{I}}{\partial y_{A}} \div \partial_{1} \frac{\partial \mathcal{I}}{\partial y_{A, t}}+\cdots\right] \text {. }
$$

\section{a) A Symmetry of and Lagrangian for the Perturbed Field Equations}

The system of linear equations (2.3) is symmetric up to a divergence in the following sense:

Proposition 1. For any two independent fields $\delta y_{A}$ and $\bar{\delta} y_{A}$

To see this, observe that

$$
\bar{\delta} y_{A} \delta F^{A}=\delta y_{A} \bar{\delta} F^{A}+\partial_{i} R^{i}
$$

$$
[\delta, \bar{\delta}]=\left[\delta, \partial_{i}\right]=\left[\delta, \frac{\partial}{\partial y_{A}}\right]=\left[\delta, \frac{\partial}{\partial y_{A, i}}\right]=0,
$$

on sufficiently smooth functions. Therefore, we have

$$
\delta F^{A}=\frac{\partial}{\partial y_{A}} \delta \mathfrak{L}-\partial_{i} \frac{\partial}{\partial y_{A, i}} \delta \mathfrak{L}+\cdots,
$$

from which follows the result

where

$$
\bar{\delta} y_{A} \delta F^{A}=\delta \bar{\delta} \varepsilon-\partial_{i} Q^{i}
$$

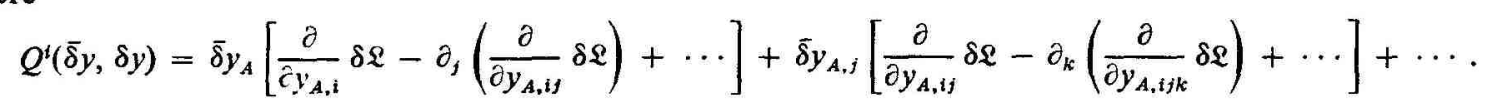

But $\bar{\delta} \delta \mathcal{Q}$ is symmetric, and Proposition 1 follows, with $R^{i}=Q^{i}(\bar{\delta} y, \delta y)-Q^{i}(\delta y, \bar{\delta} y)$. 
If for notational clarity we define the linear operator $L^{A}(\delta y) \equiv \delta F^{A}$, then the symmetry property (2.4) can be stated in the form

$$
\bar{z}_{A} L^{A}(z)=z_{A} L^{A}(\bar{z})+\partial_{t} R^{1}(z, \bar{z}),
$$

where $R^{t}$ is bilinear in the arbitrary fields, $z_{A}$ and $\bar{z}_{A}$. An associated action for the linearized equations $(2.3)$ is given by

Proposition 2. $\mathfrak{R}_{2}=z_{A} L^{A}(z)$ is a Lagrangian density for the perturbed field equations. That is, by demanding that

$$
\delta \int_{\Omega} \varepsilon_{2}=0,
$$

for all variations $\delta z_{A}$ that vanish on the boundary $\partial \Omega$, we obtain

$$
\begin{aligned}
\delta \int_{\Omega} z_{A} L^{A}(z)= & \Leftrightarrow \int_{\Omega}\left[\delta z_{A} L^{A}(z)+z_{A} L^{A}(\delta z)\right]=0 \\
& \Leftrightarrow 2 \int_{\Omega} \delta z_{A} L^{A}(z)=0, \\
& \Leftrightarrow L^{A}(z)=0 \quad \text { in the region } \Omega .^{1}
\end{aligned}
$$

\section{b) Harmonic Time Dependence}

Let us now assume that the unperturbed fields are a time-independent solution of the field equations $F^{A}=$ We want to investigate the stability of that equilibrium, to discover whether the solution $\delta y_{A}$ to the lineariz equations satisfying physical boundary conditions and corresponding to otherwise arbitrary initial data will remain bounded in time. Because $\mathfrak{E}$ has no explicit time dependence, the linear operators associated with a stationary equilibrium will also be time-independent and will therefore admit product solutions of the form $y_{A}=y_{A}\left(x^{\alpha}\right) e^{\text {tot }}$ ( $x^{\tau}$ is a point in the $t=$ const. 3-space). Our object now will be to obtain a formula for $\sigma$ and from it to derive conditions on stability.

In the case of harmonic time dependence, the operator $L^{A}$ takes the form

$$
L^{A}\left(z e^{i \sigma t}\right)=e^{i \sigma t} \sigma^{n} L_{n}{ }^{A}(z),
$$

where the operators, $L_{n}$ are alternately real and imaginary $\left(L_{0}\right.$ is real) and there is an implied sum over $n$. Then by virtue of the symmetry relation, equation (2.6), arbitrary fields $z\left(z^{a}\right), \bar{z}\left(x^{a}\right)$, and frequencies $\sigma$ satisfy

$$
e^{-i \sigma t} \bar{z}_{A} L^{A}\left(z e^{i \sigma t}\right)=e^{+i \sigma t_{B}} L^{A}\left(e^{-i \sigma t} \bar{z}\right)+\partial_{i} R^{i}(z, \bar{z}) .
$$

Because $R^{t}$ is bilinear and $L^{A}$ is independent of time, $R^{t}$ is also independent of time and $\partial_{t} R^{t}=\partial_{\alpha} R^{\alpha}$. Equation (2.8) can then be rewritten as

$$
\sigma^{n}\left[\bar{z}_{A} L_{n}{ }^{A}(z)-(-1)^{n} z_{A} L_{n}^{A}(\bar{z})=\sigma^{n} \partial_{\alpha} R_{n}{ }^{\alpha}(z, \bar{z}),\right.
$$

where $R_{n}^{t}$ is defined by analogy with equation (2.7) for $L_{n}{ }^{A}$. But equation (2.9) holds for all $\sigma$, and we infer

LEMMA 1.

$$
\bar{z}_{A} L_{n}{ }^{A}(z)=(-1)^{n} z_{A} L_{n}{ }^{A}(\bar{z})+\partial_{\alpha} R^{\alpha}{ }_{n}(z, \bar{z}) .
$$

Thus, up to a divergence, the operators $L_{n}{ }^{A}$ are each Hermitian (real and symmetric or imaginary and antisymmetric). This lemma is the key to the stability of normal modes of Lagrangian systems. It has been proven for $n \leq 2$ by Kulsrud (1968).

\section{c) A Formula for $\sigma$}

When $z e^{i t o t}$ satisfies the perturbed equations

$$
L^{A}\left(z e^{i \sigma t}\right)=0,
$$

we can obtain a useful equation for the characteristic frequency $\sigma$ from the integral relation

$$
\int_{\Omega} z_{A}^{*} e^{-i \sigma t} L^{A}\left(z e^{i o t}\right)=0
$$

${ }_{1}^{1}$ The Lagrangian $\mathscr{L}_{2}$ is that often constructed by Chandrasekhar with many coauthors (see references); the related Lagrangian $\delta \delta \mathscr{L}$ is the second variation of $\mathcal{Q}$ that has been used by Taub (1969) and Schutz (1972); cf. Whittaker (1937). 
From Lemma 1 and equation (2.5) we see that this can be written in the form

$$
\boldsymbol{\sigma}^{n} A_{n}+S=0,
$$

where $A_{n}$ are the real (by. Lemma 1) coefficients of $\sigma^{n}$ in $\bar{\delta} \delta \&$, and where

$$
S=-\int_{\delta \Omega} \cdot Q^{\alpha} n_{\alpha}
$$

(with $n_{\alpha}$ the unit normal to $\partial \Omega$ ) is in general complex. An immediate consequence of equation (2.13) is

LEMMA 2 . The characteristic frequency of any normal mode can be purely real only if the surface integral $S$ is real.

This restriction on the existence of marginally stable modes is as far as we can go without being more specific about the nature of the system whose stability we are investigating. For bounded systems, where the surface integral can be taken outside the system and made to vanish identically, Lemma 2 will not be useful. But for systems coupled to radiation of any sort, the radiation field extends to infinity and Lemma 2 becomes important. For such systems the surface integral commonly represents the energy flux across the boundary: $R$ will vanish for periodic boundary conditions on $\partial \Omega$ (standing waves) and will take the asymptotic form -io $D$ for outgoing $(D>0)$ and incoming $(D<0)$ radiation. Then the characteristic frequency of a mode with outgoing radiation can be purely real only if it vanishes. An exception to this rule is a rotating black hole where the boundary $\partial \Omega$ consists of two disjoint pieces, the horizon and the surface at infinity; the two contributions can, in principle, cancel for "superradiant" modes in which radiation as seen by locally nonrotating observers is entering the horizon but energy as defined by asymptotic observers is leaving the black hole.

In most cases of physical interest, and in particular for the relativistic perfect fluid, only first and second time derivatives occur and equation (2.13) becomes

$$
\sigma^{2} A-\sigma B-C+S=0,
$$

where $A, B$, and $C$ are the real integrals $A_{2},-A_{1}$, and $-A_{0}$ in that equation. Moreover, $A$ will generally be positive definite, corresponding to a positive kinetic energy of the perturbation, and in the cases we will treat here $S$ will be of the form $-i \sigma D$, corresponding to outgoing radiation. The frequency equation is then

$$
\sigma^{2} A-\sigma(B+i D)-C=0
$$

with $A$ and $D$ positive definite. Under these more stringent assumptions, the existence of "unstable" roots to equation (2.15) depends on the sign of $C$. That is,

LEMma 3. The equation $\sigma^{2} A-\sigma(B+i D)-C=0$ with $A \geq 0$ and $D$ positive definite has a root $\sigma_{-}$with Im $\sigma_{-}<$ 0 if and only if $C<0$.

To prove this, observe that the roots of equations (2.15) are (for $A>0$ )

$$
\sigma_{ \pm}=(Z A)^{-1}\left\{B+i D \pm\left[B^{2}+y A C-D^{2}+2 i B D\right]^{1 / 2}\right\},
$$

with imaginary parts

$$
\alpha_{ \pm} \equiv 2 A \operatorname{Im} \sigma_{ \pm}=D \pm 2^{-1 / 2}\left\{-\left(B^{2}-D^{2}+4 A C\right)+\left[\left(B^{2}-D^{2}+4 A C\right)^{2}+4 B^{2} D^{2}\right]^{1 / 2}\right\}^{1 / 2} .
$$

Then $D>0$ implies $\alpha_{+}>0$. But

$$
2 \alpha_{+} \alpha_{-}=B^{2}+D^{2}+4 A C-\left[\left(B^{2}-D^{2}+4 A C\right)^{2}+4 B^{2} D^{2}\right]=x^{2}-\left(x^{2}-16 A C D^{2}\right)^{1 / 2},
$$

where $x=B^{2}+D^{2}+4 A C$. Therefore $2 \alpha_{+} \alpha_{-}<0 \Leftrightarrow C<0$, and $\alpha_{-}<0 \Leftrightarrow C<0$, as stated. The proof for $A=0$ is trivial.

Since only one of the roots of equation (2.15) need be the characteristic frequency of a mode, Lemma 3 does not imply instability for $C<0$. To prove that the unstable root is an eigenfrequency requires a more careful examination, which we provide for the relativistic fluid in § IV.

\section{PERTURBATIONS OF PERFECT FLUIDS IN GENERAL RELATIVITY}

\section{a) Lagrangian Perturbation Formalism}

It is conventional in dealing with perturbations of a fluid to define a Lagrangian displacement $\xi^{a}$, a vector connecting fluid elements in the unperturbed configuration to the corresponding elements in the perturbed fluid. More precisely, $\xi^{a}$ is the generator of diffeomorphisms that take world lines of the unperturbed fluid into the perturbed world lines. The change in any quantity $Q$ defined on the fluid can be described in two ways: the Eulerian variation 
$\delta Q$ is the change in $Q$ at a fixed point of the manifold, whereas the Lagrangian variation $\triangle Q$ is the variation of the field with respect to a frame that is itself dragged along (with the fluid) by the displacement $\xi^{a}$. Formally, ${ }^{2}$

$$
\Delta=\delta+f_{\xi} .
$$

Changes in fluid attributes such as baryon density $n$, energy density $\epsilon$, pressure $p$, four velocity $u^{a}$, or the energymomentum tensor $T^{a b}$ are conveniently described by $\Delta$; on the other hand, changes in the field variables-in this $h_{a} \equiv \delta g_{a b}$ cas in the metric-do not vanish outside the fluid and are generally described by the Eulerian variation, In general

gauge. That is, one has the freedom, in matchion has an additional advantage: it is invariant under a change of infinitesimal displacement ${ }^{a}$ atom, in matching points of the perturbed and unperturbed spacetimes, to make an $Q$ from $\delta Q$ to $\delta Q+f_{n} Q$. But the displacement vech a transformation changes the Eulerian variation of a quantity $Q$ from $\delta Q$ to $\delta Q+\mathfrak{f}_{n} Q$. But the displacement vector $\xi^{a}$ connecting the perturbed and unperturbed world lines correspondingly changes from $\xi^{a}$ to $\xi^{a}-\eta^{a}$, so that the Lagrangian change in $Q$ is just

$$
\delta Q+£_{n} Q+£_{\xi-\eta} Q=\delta Q+£_{\xi} Q,
$$

as before. Alternatively, one can define $\Delta Q$ as the Eulerian change in $Q$ relative to the "comoving" gauge in which the Lagrangian displacement $\xi^{a}$ identically vanishes. A further freedom in the choice of Lagrangian displacement should be pointed out, namely, that $\xi^{a}+f u^{a}$ (where $f$ is any scalar field) leads to the same perturbed world lines as $\xi^{a}$. The Eulerian perturbations as well as the frequency formula we obtain will be invariant under the replace-

Consider a perfect fluid, characterized by an energy momentum part of $\xi^{a}:$ for example, by requiring $\xi^{a} u_{\alpha}=0$.

$$
T^{a b}=(\epsilon+p) u^{a} u^{b}+p g^{a b} .
$$

The unperturbed configuration is assumed to be a stationary but not necessarily axisymmetric ${ }^{3}$ solution to the gravitational field equations with perfect fluid source, supplemented by an equation of state, say $\epsilon=\epsilon(p, n)$. A Lagrangian displacement $\xi^{a}$ determines the fluid perturbations as follows. If $t^{a}$ is a vector field tangent to the
unperturbed world lines, the Eulerian unperturbed world lines, the Eulerian change in $t^{a}$ is by definition the result of its being dragged along by $\xi^{a}$,

Thus

$$
\delta t^{a}=-£_{\xi} t^{a} \text {. }
$$

The fluid four-velocity is

$$
\begin{gathered}
\Delta t^{a}=0 . \\
u^{a}=t^{a}\left(-g_{b c} t^{b} t^{c}\right)^{-1 / 2} \\
\Delta u^{a}=\frac{1}{2} u^{a} u^{b} u^{c} \Delta g_{b c}, \\
\Delta g_{b c}=h_{b c}+2 \nabla_{(b} \xi_{c)} .
\end{gathered}
$$

We are interested in a criterion for dynamical stability, and are therefore concerned with perturbations that proceed on a hydrodynamic time scale, too fast for heat transfer across a fluid element to be important. Thus we
assume that

$$
\frac{\Delta p}{p}=\gamma \frac{\Delta \epsilon}{\epsilon+p}
$$

where $\gamma$ is the adiabatic index. Finally, the perturbed equation

admits the immediate first integral

$$
\Delta\left(u_{a} \nabla_{b} T^{a b}\right)=0
$$

$$
\frac{\Delta \epsilon}{\epsilon+p}=-\frac{1}{2} q^{a b} \Delta g_{a b}=\frac{\Delta n}{n}
$$

where $q^{a b}=g^{a b}+u^{a} u^{b}$ is the projection operator orthogonal to the fluid velocity $u^{a}$, and where $\Delta n / n$ is the fractional change of baryon number, or of any conserved scalar. By introducing the baryon density $n$, one obtains a

2 This definition conforms to that used by Taub (1969) and by Carter (1973a), but agrees with that of Chandrasekhar et al. and (1967) only for scalars. The latter authors use $\Delta=\delta+\xi \cdot \nabla$.

${ }^{3}$ Whether stationary nonaxisymmetric perfect fluid configurations (analogous to the Newtonian Dedekind ellipsoids) exist in and Elbert 1974), and an implication of such solutions within the lowest order post-Newtonian approximation (Chandrasekhar figurations; presumably to these solutions correspond solutions in the exymmetric linear perturbations to marginally stable constar to represent a point of bifurcation from which a sequence of in the exact theory. That is, one expects such a marginally stable 
useful shorthand for the right-hand side of equation (3.9), which is just the Lagrangian change in the spatial volume orthogonal to the fluid world lines; and $n$ also serves as a second parameter in the equation of state. One could equally well have introduced the conserved entropy per baryon.

\section{b) The Symmetry Property}

The general results of $\S$ II followed from the symmetric character of the perturbation equations, which we proved by assuming that they were derivable as the Euler-Lagrange equations of some Lagrangian. Because the Einstein tensor is derivable from the Lagrangian $R$, we have the following result.

LEMMA 4. For arbitrary $h_{a b}=\delta g_{a b}, \bar{h}_{\mathrm{a} b}=\bar{\delta} g_{\mathrm{ab}}$,

$$
h_{a b} \delta\left[G^{a b}(-g)^{1 / 2}\right]=h_{a b} \delta\left[G_{a b}(-g)^{1 / 2}\right]+(-g)^{1 / 2} \nabla_{a} S^{a},
$$

where $S^{a}$ is bilinear in $h_{\alpha b}$ and $\bar{h}_{a b}$. In fact, using the well-known relation (see, e.g., Geroch 1972 for a concise derivation)

$$
\delta R_{a b}=\nabla^{c} \nabla_{(a} h_{b) c}-\frac{1}{2} \nabla_{a} \nabla_{b} h-\frac{1}{2} \nabla^{c} \nabla_{c} h_{a b},
$$

we find (details are given in Appendix III)

where

$$
(-g)^{-1 / 2} \delta\left[G^{a b}(-g)^{1 / 2}\right]=\frac{1}{2} \epsilon^{a c e g} \epsilon^{b d f} \nabla_{(c} \nabla_{d)} h_{e f}+G^{a b c d} h_{c d}
$$

$$
\begin{aligned}
G_{a b c d}= & \frac{1}{2} R_{a(c d) b}+\frac{1}{2}\left[R_{a(c} g_{d) b}+R_{b c c} g_{d) a}-R_{a b} g_{c d}-R_{c d} g_{a b}\right] \\
& -\frac{1}{4} R\left(g_{a c} g_{b d}+g_{a d} g_{b c}-g_{a b} g_{c d}\right), \\
= & G_{(a b)(c d)}=G_{c d a b} .
\end{aligned}
$$

Lemma 4 can be written in the form

$$
(-g)^{-1 / 2} \bar{h}_{a b} \delta\left[G^{a b}(-g)^{1 / 2}\right]=-\frac{1}{2} \epsilon^{a c e g} \epsilon_{g}^{b d f} \nabla_{c} \bar{h}_{a b} \nabla_{d} h_{e f}+G^{a b c d} \bar{h}_{a b} h_{c d}+\nabla_{c}\left(\frac{1}{2} \epsilon^{a c e g} \epsilon^{b d f} \bar{h}_{a b} \nabla_{d} h_{e f}\right) .
$$

Lagrangians exist for perfect fluids (e.g., Taub 1954; Schutz 1970), but we shall prove the symmetry property directly. The formulae of $\S$ III $a$ determine the Lagrangian change in the energy-momentum tensor $T^{a b}$, namely,

where the tensor

$$
(-g)^{-1 / 2} \Delta\left[T^{a b}(-g)^{1 / 2}\right]=W^{a b c d} \Delta g_{c d},
$$

$$
W^{a b c d}=\frac{1}{2}(\epsilon+p) u^{a} u^{b} u^{c} u^{d}+\frac{1}{2} p\left(g^{a b} g^{c d}-g^{a c} g^{b d}-g^{a d} g^{b c}\right)-\frac{1}{2} \gamma p q^{a b} q^{c d}
$$

has the symmetries $W^{a b c d}=W^{(a b)(c d)}=W^{c d a b}$. An equivalent expression appears in Carter and Quintana (1972) although there the symmetries are not apparent. From equation (3.14) follows

LEMMA 5. For arbitrarily chosen tensor fields $\xi^{a}, \bar{\xi}^{a}, h_{a b}$, and $\bar{h}_{a b}$,

Here

$$
\bar{\Delta} g_{a b} \Delta\left[T^{a b}(-g)^{1 / 2}\right]=\Delta g_{a b} \bar{\Delta}\left[T^{a b}(-g)^{1 / 2}\right] \text {. }
$$

$$
\bar{\Delta} g_{a b} \equiv \bar{\delta} g_{a b}+f_{\xi} g_{a b}=\bar{h}_{a b}+2 \nabla_{(a)} \bar{\xi}_{b)} .
$$

Lemmas 4 and 5 together imply the symmetry property of the perturbation equations. That is, LEMMA 6.

$$
16 \pi \bar{\xi}_{a} \Delta\left(\nabla_{b} T^{a b}\right)+\bar{h}_{a b} \delta\left(G^{a b}-8 \pi T^{a b}\right)=16 \pi \xi_{a} \bar{\Delta}\left(\nabla_{b} T^{a b}\right)+h_{a b} \bar{\delta}\left(G^{a b}-8 \pi T^{a b}\right)+\nabla_{a} R^{a},
$$

where the vector $R^{a}$ is bilinear in the barred and unbarred quantities and reduces to $S^{a}$ of Lemma 3 (eq. [3.10]) outside the star. The symmetry relation can be written explicitly in the form

$$
\begin{aligned}
16 \pi \bar{\xi}_{a} \Delta\left(\nabla_{b} T^{a b}\right)+\bar{h}_{a b} \delta\left(G^{a b}-8 \pi T^{a b}\right)= & -16 \pi U^{a b c d} \nabla_{a} \bar{\xi}_{b} \nabla_{c} \xi_{d}-16 \pi V^{a b c d}\left(\bar{h}_{a b} \nabla_{c} \xi_{d}+h_{a b} \nabla_{c} \bar{\xi}_{d}\right) \\
& -\frac{1}{2} \epsilon^{a c e g} \epsilon^{b d f}{ }_{g} \nabla_{c} \bar{h}_{a b} \nabla_{d} h_{e f}+8 \pi \nabla^{c} T^{a b}\left(\bar{h}_{a b} \xi_{c}+h_{a b} \bar{\xi}_{c}\right) \\
& +16 \pi T^{a b} R_{a c b d} \bar{\xi}^{c} \xi^{d}+\left(G^{a b c d}-8 \pi W^{a b c d}\right) \bar{h}_{a b} h_{c d} \\
& +\nabla_{a}\left[16 \pi U^{a b c d} \bar{\xi}_{b} \nabla_{c} \xi_{d}+16 \pi V^{c d a b} h_{c d} \xi_{b}\right. \\
& \left.+\frac{1}{2} \epsilon^{a c e g} \epsilon^{b d f}{ }_{g} \bar{h}_{c d} \nabla_{b} h_{e f}\right],
\end{aligned}
$$

where

$$
U^{a b c d}=(\epsilon+p) u^{a} u^{c} q^{b d}+p\left(g^{a b} g^{c d}-g^{a d} g^{b c}\right)-\gamma p q^{a b} q^{c d}=U^{c d a b}
$$

and

$$
V^{a b c d}=\frac{1}{2}(\epsilon+p)\left(u^{a} u^{c} q^{b d}+u^{b} u^{c} q^{a d}-u^{a} u^{b} q^{c d}\right)-\frac{1}{2} \gamma p q^{a b} q^{c d} .
$$

A derivation of the formulae in this section is provided in Appendix III. 


\section{c) A Formula for the Frequency}

We want to consider perturbations with harmonic time dependence, which can be introduced as follows. We select as a family of spacelike or null hypersurfaces in the background spacetime the images of some such arbitrarily chosen surface $S$ under group of transformations generated by the (asymptotically) timelike Killing vector $\zeta^{a}$. The perturbed field equations are a set of linear differential equations in $\xi^{a}$ and $h_{a b}$ with coefficients independent of the Killing time, which therefore admit solutions of the form

$$
\xi^{a}=\xi^{a}\left(x^{\alpha}\right) e^{i a t}, \quad h_{a b}=h_{a b}\left(x^{\alpha}\right) e^{i a t},
$$

where $x^{\alpha}$ is a point in the spatial manifold of Killing trajectories. From now on we shall use ' $\xi$ ' and ' $h a b$ ' to denote functions of $x^{\alpha}$ only, that is, tensors for which $\mathfrak{f}_{\xi} \xi^{a}=0, \mathfrak{f}_{\xi} h_{a b}=0$. In previous formulae we then make the replacements

$$
\begin{gathered}
\nabla_{a} \xi^{b} \rightarrow \nabla_{a}\left(\xi_{b} e^{i \sigma t}\right)=e^{i \sigma t}\left(\nabla_{a} \xi^{b}+i \sigma \xi^{b} \nabla_{a} t\right)=e^{i \sigma t}\left(\nabla_{a} \xi^{b}+i \sigma n_{a} \xi^{b}\right) \\
\nabla_{a} h_{b c} \rightarrow e^{-i \sigma t}\left(\nabla_{a} \bar{h}_{b c}-i \sigma n_{a} \bar{h}_{b c}\right)
\end{gathered}
$$

where $n_{a}=\nabla_{a} t$ is a vector normal to $S$.

In this way, equation (3.18) can be rewritten in the form of equation (2.14),

with

$$
\sigma^{2} \mathscr{A}-i \sigma \mathscr{B}-\mathscr{C}+\nabla_{a} \mathscr{R}^{a}=0
$$

and

$$
\begin{aligned}
\mathscr{A}= & 16 \pi U^{a b c d} n_{a} n_{c} \bar{\xi}_{b} \xi_{d}+\frac{1}{2} \epsilon^{a c e g} n_{a} \epsilon^{b d f}{ }_{g} n_{b} \bar{h}_{c d} h_{e f}, \\
\mathscr{B}= & 16 \pi U^{a b c d} n_{a}\left(\bar{\xi}_{b} \nabla_{c} \xi_{d}-\xi_{b} \nabla_{c} \bar{\xi}_{d}\right)+16 \pi V^{a b c d} n_{c}\left(h_{a b} \bar{\xi}_{d}-\bar{h}_{a b} \xi_{d}\right) \\
& +\frac{1}{2} \epsilon^{a c e g} \epsilon^{b d f}{ }_{g} n_{a}\left(\bar{h}_{c d} \nabla_{b} h_{e f}-h_{c d} \nabla_{b} \bar{h}_{e f}\right), \\
\mathscr{C}= & -16 \pi U^{a b c d} \nabla_{a} \bar{\xi}_{b} \nabla_{c} \xi_{d}-16 \pi V^{a b c d}\left(\bar{h}_{a b} \nabla_{c} \xi_{d}+h_{a b} \nabla_{c} \bar{\xi}_{d}\right) \\
& -\frac{1}{2} \epsilon^{a c e g} \epsilon^{b d f}{ }_{g} \nabla_{c} \bar{h}_{a b} \nabla_{d} h_{e f}+8 \pi \nabla^{c} T^{a b}\left(\bar{h}_{a b} \xi_{c}+h_{a b} \bar{\xi}_{c}\right) \\
& +16 \pi T^{a b} R_{a b c d} \bar{\xi}^{c} \xi^{d}+\left(G^{a b c d}-8 \pi W^{a b c d}\right) \bar{h}_{a b} h_{c d},
\end{aligned}
$$

$$
\mathscr{R}^{a}=-16 \pi U^{a b c d} \bar{\xi}_{b}\left(\nabla_{c}+i \sigma n_{c}\right) \xi_{d}-16 \pi V^{c d a b} h_{c a} \bar{\xi}_{b}-\frac{1}{2} \epsilon^{a c e g} \epsilon^{b d f}{ }_{g} \bar{h}_{c a}\left(\nabla_{b}+i \sigma n_{b}\right) h_{e f} .
$$

As implied by the discussion in $\S I I, \mathscr{A}, i \mathscr{B}$, and $\mathscr{C}$ are respectively symmetric, antisymmetric, and symmetric in the barred and unbarred quantities, and are therefore all Hermitian.

We now wish to integrate equation (3.19) over the hypersurface $S$ and in particular to allow for the case where $S$ is null. Let $\Omega$ be the volume element on $M$ (in local coordinates $\Omega=(-g)^{1 / 2} d t \wedge d x^{1} \wedge d x^{2} \wedge d x^{3}$ ). Then a volume $\boldsymbol{\Sigma}$ on $S$ is the restriction to $S$ of the three form $\Omega(\zeta)$, with $\zeta$ the Killing vector. In local coordinates $\left(t, x^{\alpha}\right)$, with $x^{\alpha}$ constant along the Killing trajectories, we have $\operatorname{dt}(\zeta)=\partial_{t} t=1$ and $d x^{\alpha}(\zeta)=0$. Then $\Sigma=(-g)^{1 / 2} d x^{1} \wedge$ $d x^{2} \wedge d x^{3}$. This is related to the surface element $d \Sigma_{a}=n_{a} \Sigma$ that appears in Gauss's law by $\Sigma=\zeta^{a} d \Sigma_{a}$, but it is not the intrinsic volume determined by the metric in $S$; this latter three-form is $\left(n_{a} n^{a}\right)^{1 / 2} \mathbf{\Sigma}$ and vanishes for null hypersurfaces. If $A^{a}$ is any time-independent vector in $M$, we have $\left(\nabla_{a} A^{a}\right) \boldsymbol{\Sigma}=d[\boldsymbol{\Sigma}(A)]$, where the forms are on $S$. Thus

$$
\int_{S} \nabla_{a} A^{a} \mathbf{\Sigma}=\int_{\partial S} \mathbf{\Sigma}(A)
$$

Now let $\bar{\xi}^{a}=\xi^{a *}, h_{a b}=h_{a b}{ }^{*}$, and assume that $\xi^{a}$ and $h_{a b}$ satisfy the perturbed field equations for $\sigma$. Then the integral over $S$ of equation (3.19) is the frequency equation

where

$$
\sigma^{2} A-\sigma B-C+R=0,
$$

$$
A=\int_{S} \mathscr{A} \mathbf{\Sigma}, \quad B=\int_{S} i \mathscr{B} \mathbf{\Sigma}, \quad \text { and } \quad C=\int_{S} \mathscr{C} \mathbf{\Sigma}
$$

are all real, and

$$
R=\int_{\partial s} \boldsymbol{\Sigma}(\mathscr{R})
$$

is complex. 


\section{TWO STABILITY THEOREMS}

We will now specialize the gauge and choose a hypersurface in such a way that $A$ becomes manifestly positive and $R$ assumes the form $i \sigma D$, with $D$ positive for outgoing radiation. Spacelike hypersurfaces have the problem that outgoing modes behave asymptotically like $h_{a b} \sim r^{k} e^{i \sigma(t-r)}$ and so stable modes $(\operatorname{Im} \sigma>0)$ grow exponentially as $r \rightarrow \infty$. This is important because $(i \sigma)^{-1} R$ becomes real only asymptotically. To keep the coefficients $A, B, C$, and $R$ finite, we therefore choose a null hypersurface; and, for convenience in describing the symptotic behavior, we assume that it is the hypersurface swept out by a flash of light emitted at the star's center of mass. The resulting family of (Killing related) null hypersurfaces will be distinguished by an index $u$ in such a way that $\zeta^{a} \Gamma_{a} u=$ $\partial_{u} u=1$.

The gauge will be specified by setting $h_{a b} n^{b}=0$. Then the last term in the integrand $\mathcal{Q}$ vanishes:

$$
\frac{1}{2} \epsilon^{a c e g} \epsilon^{b d f}{ }_{g} n_{c} n_{d} h^{*}{ }_{a b} h_{e f}=\frac{1}{2} n^{a} n_{a}\left(h^{*} h-h^{*}{ }_{b c} h^{b c}\right)=0,
$$

and the remaining term is

$$
U^{a b c d} n_{a} n_{c} \xi_{b}{ }^{*} \xi_{d}=(\epsilon+p)\left(u^{a} n_{a}\right)^{2} q^{b d} \xi_{b}{ }^{*} \xi^{d}-\gamma p q^{a b} q^{c d} n_{a} n_{c} \xi_{b}^{*} \xi_{d},
$$

which is positive-definite when the causality condition (speed of sound) $=(\epsilon+p)^{-1} \gamma p<1$ is satisfied. Hence $\mathscr{A} \geq 0$, and $s t=0$ only when $h_{a b} \xi^{b}=0$ everywhere.

We evaluate $R$ by examining the asymptotic field equations under the assumption of outgoing radiation and no horizon. With $r$ as the radial affine parameter and with $\theta$ and $\varphi$ coordinates labeling the geodesics in such a way that the background metric tends asymptotically to

$$
d s^{2}=-d u^{2}+2 d u d v+r^{2}\left(d \theta^{2}+\sin ^{2} \theta d \varphi\right)
$$

we find (see, e.g. Newman and Unti 1962)

$$
h^{\mathrm{rr}}=O\left(r^{-1}\right), \quad h^{r \theta}=O\left(r^{-2}\right), \quad h^{r \omega}=O\left(r^{-2}\right),
$$

and these will not contribute to the surface integral. The contributions to $R$ arise from the angular components which involve only the asymptotic shear (of the radial null geodesics):

and

$$
h^{\theta \theta}=2 r^{-3}\left(\operatorname{Re} s_{1}+i \operatorname{Re} s_{2}\right)+O\left(r^{-4}\right)=-h^{\varphi \varphi} \sin ^{2} \theta
$$

$$
h^{0 \omega}=2 r^{-3}\left(\operatorname{Im} s_{1}+i \operatorname{Im} s_{2}\right),
$$

where $s_{1} r^{-2}$ and $s_{2} r^{-2}$ are the asymptotic shears of the null geodesics in the physical solutions $\operatorname{Re} h^{a b}$ and $I m h^{a b}$. Then

$$
R=\int_{c S} \Sigma(\mathscr{R})=\int \mathscr{R} r^{2} \sin ^{2} \theta d \theta d \varphi=-4 i \sigma \int\left(\left|s_{1}\right|^{2}+\left|s_{2}\right|^{2}\right) \sin ^{2} \theta d \theta d \varphi .
$$

Thus $R=-i \sigma D$, where $D \geq 0$. Because $\left|s_{1}\right|^{2}$ and $\left|s_{2}\right|^{2}$ are the Bondi news functions of $\operatorname{Re} h_{a b}$ and Im $h_{a b}$, they can vanish only when a mode is nonradiative.

The frequency equation is therefore of the form discussed in $\S \mathbf{H}$, and an immediate consequence is that the characteristic frequency of a radiative mode can be purely real only if it vanishes. Nonradiating modes can occur for radial pulsations of spherical stars and [to $O\left(\Omega^{2}\right)$ ] for quasi-radial pulsations of slowly rotating stars, but in these cases the term linear in $\sigma$ in the frequency equation vanishes and the following theorem remains valid.

THEOREM 1 (Weak Stability Theorem). If the normal modes and their characteristic frequencies of oscillation vary smoothly along a sequence of equilibrium configurations, then instability to any mode can set in only at a point along the sequence where its frequency vanishes.

Proof. By Lemma 3, unstable modes occur only when $C<0$ and in that case correspond to the root

$$
\sigma_{-}=(2 A)^{-1}\left\{B+i D-\left[B^{2}-4 A C-D^{2}+2 i B D\right]^{1 / 2}\right\} \text {. }
$$

Along the stable part of the sequence, Im $\sigma_{-}$must be positive, which implies that $C$ will also be positive. Smoothness of the eigenfunctions then ensures that the mode becomes unstable through $C=0$, whence $\sigma_{-}=0$ as well.

This theorem considerably simplifies the problem of deciding which stars are stable in general relativity. One need never consider complex frequencies or radiative modes, and it is therefore not necessary in stability calculations to use finite regions of integration or null hypersurfaces in order to avoid divergent expressions. In particular, the following result holds.

THEOREM 2 (Strong Stability Theorem). Suppose as before that the normal modes and their characteristic frequencies vary smoothly along a sequence of equilibrium configurations indexed by some parameter $e$. Consider the set of all time-independent trial functions $h_{a b}$ and $\xi^{a}$ satisfying the initial value equations ${ }^{4} \delta G^{(0)(a)}=8 \pi \delta T^{(0)(a)}$

\footnotetext{
${ }^{4}$ We are using the convention that indices in parenthesis are components along an orthonormal tetrad. The particular tetrad
} here is arbitrary. 
in any convenient gauge consistent with the asymptotic behavior $h_{(a)(b)}=O\left(r^{-1}\right)$. Then,

1) If the functional $C$ associated with some equilibrium configuration is positive for all such trial functions, all perturbations of the configuration are stable.

2) If the greatest lower bound for all normalized trial functions ${ }^{5}$ of $C$ is positive along a sequence of equilibrium configurations, until some point $e_{0}$, and if it passes through zero at that point and becomes negative for the subsequent configurations, then instability sets in at $e_{0}$.

3) The stability criterion of (1) and (2) is independent of the particular spacelike or null hypersurface chosen and of the gauge to within the demand of regular asymptotic behavior.

We will first prove the last statement, that $C$ is invariant under a (gauge) transformation of the form $h_{a b} \rightarrow h_{a b}+$ $2 \nabla_{(a} \eta_{b)}, \xi^{a} \rightarrow \xi^{a}+\eta^{a}$ when the orthonormal components of $h_{a b}$ and $\eta^{a}$ are time independent and asymptotically
$O\left(r^{-1}\right)$. When $\sigma$ vanishes, equation (3.21) reduces to

Under

$$
C=\int_{S}\left[h^{*}{ }_{a b} \delta\left(G^{a b}-8 \pi T^{a b}\right)+8 \pi \xi_{a}^{*} \Delta\left(\nabla_{b} T^{a b}\right)\right] \Sigma
$$

$$
\begin{aligned}
h_{a b} \rightarrow h_{a b}+2 \nabla_{(a} \eta_{b)}, & \\
C \rightarrow C+2 \operatorname{Re}\left\{\int_{S}\left[-\nabla_{a} \eta_{b}{ }^{*} \delta\left(G^{a b}-8 \pi T^{a b}\right)+8 \pi \eta_{a}{ }^{*} \delta\left(\nabla_{b} T^{a b}\right)\right] \boldsymbol{\Sigma}\right\} & \\
& =C+2 \operatorname{Re}\left\{\int_{S} \eta_{a}^{*}\left[\delta \nabla_{b} G^{a b}\right] \mathbf{\Sigma}+\int_{\partial S} \boldsymbol{\Sigma}\left(\eta_{a}{ }^{*} \delta G^{a b}\right)\right\}=C,
\end{aligned}
$$

where only the unperturbed field equations, the Bianchi identities, and the fact that, at large $r, \delta G_{(a)(b)} \sim r^{-3}$ fo time-independent fields $h_{a b}$ have been used. Then (1) follows from the fact that the positive-definite character of $C$ (the energy criterion of Schutz 1972). value equations do not formally in . But on any hypersurface one can always choose a gauge in which the initial value equations do not formally involve time derivatives and which is asymptotically regular. ${ }^{6}$ In that gauge positive-dalitions functions. As a result, the positive-definiteness of $C$ for time-independent trial functions is also sufficient for the stability of the star. The

that violate the equations and make $C$ negative (cf. Schutz 1972).

$e_{0}$ and negative-definite independent trial functions by

$$
C(\bar{\xi}, \xi)=\int_{S} \bar{\xi}^{*} \mathfrak{s} \boldsymbol{\Sigma}
$$

the zero frequency limit of the field equations is $\mathfrak{C} \xi=0$. Prior to $e_{0}$ all characteristic values of the operator are positive, but at any point $e_{0}+\delta e$ beyond $e_{0}$ at least one has become negative. Therefore, at some point between $e_{0}$ and $e_{0}+\delta e$, the corresponding eigenfunction is a zero frequency mode of the system.

It is not obvious that this real mode becomes unstable, however, because beyond $e_{0}$ the time-independent eigenfunctions of $\mathfrak{E}$ are not necessarily related to the time-dependent eigenfunctions of the physical system. To see that it is nevertheless true, observe that if $C=\int_{S} \eta^{*} \mathfrak{V}_{\eta}$ is evaluated on a null hypersurface, its value for any mode varies smoothly along the sequence; at a nearby point, $e_{0}+\delta e, C$ will be negative for the trial function $\eta_{t}\left(e_{0}+\delta e\right)$ which differs by $O(\delta e)$ from the real eigenfunction $\eta\left(e_{0}+\delta e\right)$. We have

$$
C_{e_{0}+\delta e}\left[\eta\left(e_{0}+\delta e\right)\right]=C e_{0}\left[\eta\left(e_{0}+\delta e\right)\right]+\left(C_{e_{0}+\delta e}-C_{e_{0}}\right)\left[\eta\left(e_{0}\right)\right]+O\left[(\delta e)^{2}\right]=C_{e_{0}+\delta e}\left[\eta_{t}\left(e_{0}+\delta e\right)\right]+O\left[(\delta e)^{2}\right]<0,
$$

where we have used the fact that $C_{e_{0}}$ is an extremum for $\eta\left(e_{0}\right)$ to show that $C_{e_{0}}\left[\eta\left(e_{0}+\delta e\right)\right]$ was $O\left[(\delta e)^{2}\right]$. In other words, the value of $C$ for the true eigenfunction will be negative just beyond $e_{0}$, which implies that the mode becomes unstable there. We have shown that the sequence becomes unstable before $e_{0}+\delta e$ for arbitrarily small $\delta e$; hence,
instability sets in at $e_{0}$.

The gauge independence of the criterion follows from that of $(c)$ discussed above. The independence of hyper$C$ is a scalar on the spatial that when evaluated on the set of time-independent trial functions, the integrand in $C$ is a scalar on the spatial manifold of Killing trajectories; the volume element is similarly interpretable as a

"The normalization can be arbitrary as long as it is positive-definite. This excludes the "natural" normalization $A=1$ since $A$ is only positive-semidefinite on a null hypersurface; but $A$ can be used if one uses a spacelike hypersurface that is only asymptotically null, on which a gauge can always be chosen in which $A$ is positive-definite.

${ }^{6}$ This can be seen by writing $\delta G^{0 a}=\partial_{t}\left[L_{1}{ }^{0 a}(\xi)+L_{2}{ }^{0 a}(h)\right]+L_{3}{ }^{0 a}(\xi)+L_{4}{ }^{0 a}(h)$. The gauge conditions are $\partial_{t}\left[L_{1}{ }^{0 a} \xi+L_{2}{ }^{0 a}(h)\right]=$
and the initial value equations become $L_{3}{ }^{0 a}(\xi)+L_{4}{ }^{\circ a}(h)=0$ $\left.h_{B}{ }^{g}, \alpha\right]=0$ while the initial value equations become $h_{0 \alpha}=0$. 
volume on the manifold of trajectories because its definition involved only the Killing vector, not the normal to a particular hypersurface.

In order to use Theorem 2 , one must know that $C$ is positive-definite somewhere along the sequence. In Appendix I we prove that $C$ is positive-definite for Newtonian stars provided they satisfy the Newtonian secular stability criterion (Lynden-Bell and Ostriker 1967), which is simply that the Newtonian part of $C$ be positive. The proof effectively shows that the gravitational wave parts of $C$ do not affect the stability of secularly stable Newtonian stars. This result is not restricted to perfect fluids: any Newtonian configuration is stable against gravitational radiation if and only if the Newtonian part of $C$ is positive-definite. ${ }^{7}$ Thus since secular and dynamical stability are identical for nonrotating stars, one can take each sequence in Theorem 2 to originate at a stable nonrotating Newtonian star.

We should note that Theorems 1 and 2 apply immediately to any system in general relativity provided that (i) the coefficient $A$ can be made positive-definite on a null hypersurface, and (ii) either the system is bounded, so that the surface integral involves only gravitational radiation, or any extra radiation terms preserve the sign of the surface integral. That elastic solids and electromagnetism satisfy these conditions is shown in Appendix II. Continuity of the eigenfrequencies may be difficult to prove in general, but is not likely to fail under reasonable conditions; it has been proved for Kerr by Hartle and Wilkins (1974).

Finally, it should be pointed out that for radiative modes, instability defined by the existence of modes with Im $\sigma<0$ has a clear physical meaning, again independent of gauge. The outgoing energy, defined by means of the Bondi news function (which, as we observed, appears as the surface term of the null hypersurface frequency equation), grows without limit for unstable modes, with growth rate $\mathrm{Im} \sigma$, and dies off for stable modes. It is independent of gauge transformations that conform asymptotically to the BMS group (Sachs 1962), because under such a transformation the change in the news is of higher order in the perturbation; and so Im $\sigma$ represents an unambiguous growth rate of the system's radiated energy. The characteristic frequencies are similarly definable as the set of pure frequencies admissible as the time dependence of the news function.

In proving the strong stability theorem, we assumed that the eigenfunctions of the Hermitian operator $\mathfrak{G}$ were complete for the set of time-independent trial functions. We did not, however, make any assumptions about completeness of the outgoing normal modes of the system, only requiring that they vary smoothly along the sequence. In classical problems of fluid mechanics with shear (see, e.g., Chandrasekhar 1961), these latter modes are not strictly complete because the perturbation equations can have singular points when the pattern speed $\sigma / m$ is equal to the angular velocity $\Omega$ (with $m$ the axial eigenvalue). In such cases a complete set must include functions whose first derivatives are discontinuous at the singular point. In general relativity the outgoing normal modes may be complete in any event, because when the frequencies are complex, such singular points do not arise.

\section{v. DISCUSSION AND CONCLUSION}

The theorems here show that (at least for configurations satisfying our assumptions of smoothness and completeness) the generic instability of rapidly rotating stars is analogous to the "Dedekind" mode in the classical theory. It sets in when a star can be adiabatically deformed to a nearby nonaxisymmetric configuration that is stationary with respect to an observer at infinity. In the Newtonian limit (see Appendix) the frequency equation reduces to the expression of Lynden-Bell and Ostriker (1967) or, in the time-independent case, to the related expression of Chandrasekhar and Lebovitz (1973) (depending on whether the initial value equations have been formally solved). The condition $C=0$ then becomes the Newtonian criterion for secular stability which Ostriker and co-workers have found to be approximated for a wide range of differentially rotating stars by $t \equiv T /|W| \leqslant$ 0.14 , where $T$ and $W$ are the rotational kinetic energy and the potential energy, respectively. (A star is secularly unstable when nearby lower energy configurations exist but are accessible only in the presence of some dissipative mechanism, such as radiation or viscosity, which operate on longer than hydrodynamic time scales.)

Because we assumed only that the equilibrium configuration was stationary, the theorems are valid for nonaxisymmetric perfect fluid equilibria, should they exist in the exact framework of general relativity as they do in the classical theory. These could be stable for larger values of $t$, although in the known cases of uniform-density classical stars, the nonaxisymmetric sequences are unstable by $t \sim 0.20$. (This can be calculated from results in Chandrasekhar 1969.) The existence of zero-frequency nonaxisymmetric modes already means that there are stationary nonaxisymmetric solutions to the equations linearized about an axisymmetric star.

One consequence of the generic instability is to forbid configurations with the extreme differential rotation that would be required substantially to raise the mass limits on neutron stars or white dwarfs. The growth rate for nearly Newtonian stars can be estimated from Chandrasekhar's (1970) expression for the instability in Maclaurin spheroids; in terms of the period $T$ of the star and the critical value $t_{c}$ of the parameter $t$ at which instability sets in, one can show that the growth time $\tau$ is given by

$$
\frac{\tau}{T} \sim 10^{-7}\left(\frac{R \Omega}{c}\right)^{-5}\left(t-t_{\mathrm{c}}\right)^{-5}
$$

\footnotetext{
7 Although formally a secularly unstable but dynamically stable Newtonian configuration is unstable against gravitation radiation, the time scale for the growth of this instability may be much longer than the age of the Universe. Time scales are discussed in the final section.
} 
Typical times for neutron stars range from milliseconds to months (cf. Friedman and Schutz 1975). Times for white dwarfs are substantially longer, but probably still short enough to rule out exotic dwarf or neutron star models of the high-mass compact component of Cygnus X-1 (Brecher and Morrison 1973). In fact, massive neutron stars with high differential rotation would more probably represent an avenue to black hole formation than a way of avoiding collapse. That is, neutron stars which form with rotational energies limited only by the requirement of Newtonian dynamical stability $t \leqslant 0.27$ would normally radiate their angular momentum gravitationally until the point of secular stability was attained. If, however, their masses were close to the limit allowed by the initial rotation, then the spin-down would lead first to a pulsational instability. And in such a case, it seems unlikely that enough energy would be available to prevent collapse. ${ }^{8}$

The numerical work needed to locate in general relativity a rotational boundary analogous to the Newtonian $t \sim 0.14$ is still to be done. We are, however, able to show that for any star with an ergo-region the stability functional $C$ can always be made negative (Friedman 1975). Thus instability to a nonaxisymmetric mode always sets in along a sequence of rotating stars before the appearance of an ergo-region. ${ }^{9}$ For highly relativistic stars (Wilson 1972; Bardeen and Wagoner 1971; Ipser and Butterworth 1975), ergo-regions are known to occur prior to breakup velocity, and they thereby set an upper limit on rotation.

We want to thank James Bardeen, James Hartle, James Ipser, and S. Chandrasekhar for helpful discussions.

\section{APPENDIX I}

\section{STABILITY OF NEWTONIAN STARS AGAINST GRAVITATIONAL RADIATION}

In the case of a nearly Newtonian star, we will show that the functional $C$ governing stability in general relativity is positive-definite for all trial functions if and only if the star is secularly stable in the Newtonian theory. In pa ticular, the stability criterion of Theorem 2 is identically the Newtonian condition for secular stability (LyndenBell and Ostriker 1967; Chandrasekhar and Lebovitz 1973) when the perturbations are also Newtonian. This generalizes to arbitrary Newtonian stars the analogous result obtained by Chandrasekhar (1970) for the uniformdensity, uniformly rotating Maclaurin spheroids.

We want to prove that

$$
C=: \int_{S} \mathscr{C} \mathbf{\Sigma}
$$

is positive-definite for all perturbations of a secularly stable Newtonian star that obey the time-independent initial value equations. The proof shows essentially that the integrand $\mathscr{C}$ can be written as a sum of two groups of terms; the first group is just the analogous Newtonian variational expression, while the second represents a contribution of the same order arising from gravitational waves on a flat background. The integral of the first terms will be positive-definite exactly when the Newtonian star is secularly stable, whereas the second group will always be positive, reflecting the stability of flat space.

i) We first observe that when the initial value equations

$$
\delta G^{0 a}=8 \pi \delta T^{0 a}
$$

are satisfied, the functional $C$ is independent of the conjugate variables $h_{0 a}$, because the variation of $C$ with respect to arbitrary variations in $h_{0 a}$ then vanishes:

$$
\frac{\delta C}{\delta h_{0 a}}=\delta G^{0 a}-8 \pi \delta T^{0 a}=0
$$

in the time-independent case we are considering. Here $\delta C / \delta h_{0}{ }^{a}$ is a variational derivative, because the surface integral vanished for permissible trial functions. We will use our freedom in choosing $\xi^{a}$ (see $\S$ III $a$ ) to set $\xi^{0}=0$; thus only $\xi^{\alpha}$ and $h_{\alpha \theta}$ will appear in $C$.

We will subsequently make further use of the initial value equation

$$
\begin{aligned}
\delta G_{00}-8 \pi \delta T_{00}= & -\frac{1}{2} \epsilon^{\alpha \mu \nu} \epsilon^{\beta \nu}{ }_{\nu} \nabla_{\nu} \nabla_{\mu} h_{\alpha \beta}-16 \pi V^{00 c d} \nabla_{c} \xi_{d} \\
& +\left(G^{00 c d}-8 \pi W^{00 c d}-16 \pi g^{0 c} T^{0 d}\right) h_{c d}+8 \pi \xi^{c} \nabla_{c} T^{00} \\
= & 0 .
\end{aligned}
$$

A similar collapse resuiting from the spin-down of carbon-burning stars was recently suggested by Wheeler and Rosenwald (1973).

9 A proviso should be made-namely, that instability is implied only under the assumption that any time-dependent nonaxisymmetric perturbation of an axisymmetric background will be radiative. It seems apparent that any such perturbation will alter the multipole moments. 
ii) The elimination of post-Newtonian terms. The functional $C$ now has the form

$$
\begin{aligned}
C=\int_{S}\{ & -16 \pi U^{\alpha \beta \gamma \delta} \nabla_{\alpha} \xi_{\beta}{ }^{*} \nabla_{y} \xi_{\delta}-16 \pi V^{\alpha \beta \gamma \delta}\left(h_{\alpha \beta}{ }^{*} \nabla_{y} \xi_{\delta}+h_{\alpha \beta} \nabla_{y} \xi_{\delta}^{*}\right)-\frac{1}{2} \epsilon^{\alpha \mu \delta} \epsilon^{\beta \nu \gamma} \nabla_{u} h_{\alpha \beta}{ }^{*} \nabla_{\nu} h_{\gamma \delta} \\
& \left.+8 \pi \nabla_{\gamma} T^{\alpha \beta}\left(h_{\alpha \beta}{ }^{*} \xi_{y}+h_{\alpha \beta} \xi^{\gamma *}\right)+16 \pi T^{\alpha b} R_{\alpha \gamma b \delta} \xi^{\gamma *} \xi^{\delta}+\left(G^{\alpha \beta \gamma \delta}-8 \pi W^{\alpha \beta \gamma \delta}\right) h_{\alpha \beta}{ }^{*} h_{\gamma \delta}\right\} \\
= & \int_{S}\left\{\mathscr{C}_{1}+\mathscr{C}_{2}+\mathscr{C}_{3}+\mathscr{C}_{4}+\mathscr{C}_{5}+\mathscr{C}_{6}\right\} .
\end{aligned}
$$

In the unperturbed star, velocities are small: $v \ll 1$. In terms of $v$ we have $p / \rho=O\left(v^{2}\right)$ and the gravitational potential $\nu=O\left(v^{2}\right)$. In the limit of a Newtonian background, the dominant terms in $C$ are $O\left(v^{4}\right)$. We find $C_{2}, C_{4}$, and $C_{6}$ are $O\left(v^{6}\right)$ and can be neglected in comparison to $C_{1}, C_{3}$, and $C_{5}$ in which only the leading terms are retained. In equation (A2), the third term is negligible.

iii) The separation of waves from the ordinary Newtonian perturbation. The metric to Newtonian order is

$$
d s^{2}=-(1+2 \nu) d t^{2}+(1-2 \nu)\left(d x^{2}+d y^{2}+d z^{2}\right)
$$

so a strictly Newtonian perturbation would have

$$
h_{\alpha \beta}=-2 \delta \nu g_{\alpha \beta}, \quad \nabla^{2} \delta \nu=4 \pi \delta \rho .
$$

We therefore separate this part explicitly by defining

$$
h_{\alpha \beta}=K_{\alpha \beta}-2 g_{\alpha \beta} \delta \nu, \quad \nabla^{2} \delta \nu=4 \pi \delta \rho .
$$

Then $C$ can be written as the sum of three pieces:

$$
\begin{aligned}
& C=C_{\mathrm{W}}+C_{I}+C_{N} \\
& C_{W}=\int_{S}-\frac{1}{2} \epsilon^{\alpha \mu \delta} \epsilon^{\beta \nu \nu} \nabla_{\mu} K_{\alpha \beta}{ }^{*} \nabla_{\nu} K_{\gamma \delta} \\
& C_{I}=\int_{S} 2 \nabla_{\beta}\left(K^{\alpha \beta *}-g^{\alpha \beta} K^{\nu}{ }^{*}\right) \nabla_{\alpha} \delta \nu+\text { (complex conjugate) } \\
& C_{N}=\int_{S}-4|\nabla \delta \nu|^{2}-16 \pi \rho|u \cdot \nabla \xi|^{2}+16 \pi \gamma p|\nabla \cdot \xi|^{2}+16 \pi \rho \xi^{\alpha *} \xi^{\beta} \nabla_{\alpha} \nabla_{\beta} \nu-16 \pi p\left(|\nabla \cdot \xi|^{2}-\nabla_{\alpha} \xi^{\beta *} \nabla_{A} \xi^{\alpha}\right)
\end{aligned}
$$

The functional $C_{W}$ is all one has for perturbations of flat space, and is positive-definite when the flat-space initial value equations are satisfied (as we show below). The term $C_{I}$ will be shown to vanish. The functional $C_{N}$ is equal, after integrations by parts, and use of the definition of $\delta v$, to the standard Newtonian functional found, for example, in Clement (1964), Lynden-Bell and Ostriker (1967), and Chandrasekhar and Lebovitz (1973):

$\frac{1}{16 \pi} C_{N}=\int_{S} \frac{1}{2}\left(\delta \rho^{*} \delta \nu+\delta \rho \delta \nu^{*}\right)+\gamma p|\nabla \cdot \xi|^{2}+\left(\xi^{\alpha *} \nabla \cdot \xi+\xi^{\alpha} \nabla \cdot \xi^{*}\right) \nabla_{\alpha} p+\xi^{* \alpha} \xi^{\beta}\left(\rho \nabla_{\alpha} \nabla_{\beta} \nu+\nabla_{\alpha} \nabla_{\beta} p\right)-\rho|u \cdot \nabla \xi|^{2}$

iv) The positive-definiteness of $C$. We must use equation (A2), which has the form

$$
-\frac{1}{2}\left(\nabla^{2} h^{\alpha}{ }_{\alpha}-\nabla_{\alpha} \nabla_{\beta} h^{\alpha \beta}\right)+8 \pi \nabla \cdot \xi+8 \pi \xi \cdot \nabla_{\rho}=0
$$

Substituting $K_{\alpha \beta}$ into this and using the equations

gives

$$
\begin{gathered}
\delta \rho=-\nabla \cdot(\rho \xi), \quad \nabla^{2} \delta \nu=4 \pi \delta \rho, \\
\nabla^{\alpha} \nabla^{\beta}\left(K_{\alpha \beta}-g_{\alpha \beta} K^{\gamma}{ }_{\gamma}\right)=0 .
\end{gathered}
$$

This is the form the initial value equation takes in flat space, and it implies that $C_{I}$ is a divergence. Therefore, the stability problem separates completely into the stability of flat space plus the Newtonian stability of the Newtonian star.

The stability of flat space follows from the positive-definiteness of $C_{W}$ : after two integrations by parts we have

$$
2 C_{\mathrm{W}}=\int_{S}-\nabla_{\beta} K_{\gamma}^{\gamma}{ }_{\gamma}^{*} \nabla^{\beta} K_{\alpha}^{\alpha}-2 \nabla_{\beta} K_{\alpha}^{\beta *} \nabla_{\alpha} K^{\alpha \gamma}+\nabla_{\beta} K_{\alpha}^{\alpha}{ }_{\alpha} \nabla_{\gamma} K^{\gamma \beta}+\nabla_{\beta} K_{\alpha}^{\alpha} \nabla_{\gamma} K^{\gamma \beta *}+\nabla_{\alpha} K^{*}{ }_{B \gamma} \nabla^{\alpha} K^{\beta \gamma}
$$


Multiplying equation (A7) by $K^{* \mu}{ }_{\mu}$, integrating it by parts, and adding it and its complex conjugate to $2 C_{W}$ gives

$$
2 C_{W}=\int_{S} \nabla_{\alpha} K_{\mu \nu}{ }^{*} \nabla^{\alpha} K^{\mu \nu}+\nabla_{\alpha} K^{\nu}{ }_{\nu}{ }^{*} \nabla^{\alpha} K^{\mu}{ }_{\mu}-2 \nabla_{\beta} K_{\alpha}{ }^{\beta *} \nabla_{\gamma} K^{\alpha \gamma} .
$$

This is manifestly positive-definite for the gauge $\nabla_{\beta} K_{\alpha}{ }^{\beta}=0$, which we are free to choose. In fact, however, following Araki (1959) one can use Fourier transforms to show that $C_{W}$ is positive in any gauge.

Since $K_{\alpha \beta}$ and $\xi_{\alpha}$ are completely independent, we have the following result: a Newtonian star is stable in general relativity if $C_{N}$ is positive-definite, i.e., if it satisfies the Newtonian secular stability criterion. From the derivation it is clear that this result applies to all Newtonian solutions: all Newtonian equilibria secularly stable in Newtonian theory are stable against gravitational radiation.

\section{APPENDIX II \\ EXTENSIONS}

The formalism and theorems developed in this paper can be simply extended to more general Lagrangian systems. As examples, we outline the treatment of source-free electromagnetic fields and of elastic solids.

Electromagnetic fields are characterized by a stress energy tensor

$$
T_{a b}=F_{a c} F_{b}^{c}-\frac{1}{4} g_{a b} F_{c d} F^{c d} .
$$

When the perturbed field is written in terms of a perturbed vector potential,

$$
\delta F_{a b}=\nabla_{a} \delta A_{b}-\nabla_{b} \delta A_{a},
$$

the symmetry property analogous to equation (3.16) is

$$
\begin{aligned}
2 \bar{\delta} A^{a} \delta\left(\nabla^{b} F_{a b}\right)-\bar{h}^{a b}(-g)^{-1 / 2} \delta\left[T_{a b}(-g)^{1 / 2}\right] & \\
= & g^{a c} g^{b d} \bar{\delta} F_{a b} \delta F_{c d}-F_{a b c d} h^{a b} h^{c d}-2 F_{b}^{c}\left(h^{a b} \delta F_{a c}+h^{a b} \bar{\delta} F_{a c}\right) \\
& +\frac{1}{2} F^{a b}\left(\bar{h} \delta F_{a b}+h \bar{\delta} F_{a b}\right)+\nabla_{a}\left[\delta A^{b}\left(2 \delta F_{b}^{a}+2 h^{a c} F_{b c}+2 h_{b c} F^{a c}+h F^{a}{ }_{b}\right)\right], \\
F_{a b c d} & =2 F_{c(a} F_{b) d}+\frac{1}{2} g_{a b} F_{c e} F_{d}{ }^{e}+\frac{1}{2} g_{c d} F_{a e} F_{b}{ }^{e}-\frac{1}{8}\left(g_{a c} g_{b d}+g_{a d} g_{b c}+g_{a b} g_{c d}\right) F_{e f} F^{e f} \\
\quad & F_{(a b)(d)}=F_{c d a b} .
\end{aligned}
$$

The contributions to $\mathscr{A}, \mathscr{B}, \mathscr{C}$, and $\mathscr{R}^{a}$ of equation (3.19) are

$$
\begin{aligned}
\mathscr{A}_{\mathrm{EM}}= & 2\left(n^{a} n^{b}-g^{a b} n^{c} n_{c}\right) \bar{\delta} A_{a} \delta A_{b}, \\
\mathscr{O}_{\mathrm{EM}}= & 4\left[\delta A^{a} n^{b} \nabla_{[b} \bar{\delta} A_{a]}-\bar{\delta} A^{a} n^{b} \nabla_{[b} \delta A_{a]}\right. \\
& -2\left[n_{a} F_{b c}\left(\bar{h}^{a b} \delta A^{c}-h^{a b} \bar{\delta} A^{c}\right)-n_{c} F^{b c}\left(\bar{h}_{a b} \delta A^{a}-h_{a b} \bar{\delta} A^{a}\right)\right]+F^{a b} n_{a}\left(\bar{h} \delta A_{b}-\bar{h} \delta A_{b}\right), \\
\mathscr{C}_{\mathrm{EM}}= & -F_{a b c d} h^{a b} h^{c d}+4 \nabla_{[a} \bar{\delta} A_{b]} \nabla^{a} \delta A^{b}-4 F_{b}^{c}\left(h^{a b} \nabla_{[a} \delta A_{c]}+h^{a b} \nabla_{[a} \delta A_{c]}\right)+F^{a b}\left(\bar{h} \nabla_{a} \delta A_{b}+h \nabla_{a} \bar{\delta} A_{b}\right), \\
& \mathscr{R}_{\mathrm{EM}}{ }^{a}=-\bar{\delta} A^{b}\left(2 \delta F^{a}{ }_{b}+2 h^{a c} F_{b c}+2 h_{b c} F^{a c}+h F_{b}^{a}\right) .
\end{aligned}
$$

Here the vector potential is $\delta A_{a}\left(x^{\alpha}\right) e^{i \sigma t}$, and we define $\delta A^{a} \equiv g^{a b} \delta A_{b}$.

When $n^{a}$ is timelike, $\mathscr{N}_{\mathrm{EM}}$ is positive-definite. When $n^{a}$ is null, $\mathscr{A}_{\mathrm{EM}}$ is positive or zero, and can be made identcally zero by the gauge choice $n^{a} \delta A_{a}=0$. Then if one is studying only the source-free Einstein-Maxwell equations, $\mathscr{A}$ will vanish completely on the null hypersurface. The frequency equation becomes trivial, and Lemma 3 still holds for the single root of the equation. The stability theorems then also hold true if $R=\int_{\partial S} R^{\alpha} n_{\alpha}$ is still positive, which we now prove. We assume that the unperturbed electromagnetic field goes to zero at infinity at least as fast as $r^{-2}$, so that equa-
tion (A15) implies

$$
R_{E M}=\lim _{r \rightarrow \infty} 2 \int \delta A^{b *} \delta F^{r}{ }_{b} r^{2} \sin \theta d \theta d \varphi
$$

on the hypersurface $u=$ const. The gauge $n^{a} \delta A_{a}=0$ implies $\delta A_{r}=O\left(r^{-2}\right)$ which would have been the consequence of the equation $\delta\left(\nabla_{o} F^{u b}\right)=0$ anyway, had we not chosen a gauge. The remaining components $\delta A^{(a)}$ in an orthonormal basis are $O\left(r^{-1}\right)$. Then $\mathscr{R}_{\mathrm{EM}}$ becomes

$$
\mathscr{R}_{\mathrm{EM}}=-\lim _{r \rightarrow \infty} 2 i \sigma r^{2} \int\left(\left|\delta A^{(\theta)}\right|^{2}+\left|\delta A^{(\varphi)}\right|^{2}\right) \sin \theta d \theta d \varphi .
$$


This has the correct sign, so electromagnetic fields also obey Theorem 2.

For the case of an elastic solid, Carter and Quintana (1972) and Carter (1973a) give an energy momentum tensor

with

$$
T^{a b}=\epsilon u^{a} u^{b}+p^{a b},
$$

Then

$$
p^{a b}=p^{(a b)} \quad \text { and } \quad p^{a b} u_{b}=0 .
$$

with

$$
(-g)^{-1 / 2} \Delta\left[T^{a b}(-g)^{1 / 2}\right]=W^{a b c d} \Delta g_{c d}
$$

$$
W^{a b c d}=\frac{1}{2} \epsilon u^{a} u^{b} u^{c} u^{d}-\frac{1}{2} E^{a b c d}+\frac{1}{2}\left[p^{a c} u^{b} u^{d}+p^{a d} u^{b} u^{c}+p^{b c} u^{a} u^{d}+p^{b d} u^{a} u^{c}-p^{a b} u^{c} u^{d}-p^{c d} u^{a} u^{b}\right] .
$$

The elasticity tensor $E^{a b c d}$ is defined by

Because the symmetries

$$
E^{a b c d}=-2 \frac{\partial p^{a b}}{\partial q_{c d}}-p^{a b} q^{c d}=E^{(a b)(c d)}=E^{c d a b}
$$

$$
W^{a b c d}=W^{(a b)(c d)}=W^{c d a b}
$$

still hold, the equations in the body of the paper are correct as written, with $W^{a b c d}$ given by equation (A20), with

and with

$$
U^{a b c d}=2 W^{a b c d}+T^{a c} g^{b d},
$$

$$
V^{a b c d}=W^{a b c d}+\frac{1}{2}\left(T^{a c} g^{b d}+T^{b c} g^{a d}-T^{a b} g^{c d}\right) .
$$

In particular the elastic solid contribution to the coefficient of $\sigma^{2}$ is

with

$$
\mathscr{A}_{\mathrm{ES}}=U^{\mathrm{abcd}} n_{a} n_{c} \xi_{b}^{*} \xi_{d}=\left(u^{c} n_{c}\right)^{2}\left(\epsilon q^{a b}+p^{a b}\right) \xi_{a}^{*} \xi_{b}-Q^{a b} \xi_{a}^{*} \xi_{b}
$$

$$
Q^{a c}=\left(E^{a b c d}-q^{a c} p^{b d}\right) n_{b} n_{d} .
$$

But the sound velocities in the direction of propagation $q^{a b} n_{b}$ are (Carter 1973b) the eigenvalues $v$ of

$$
\left[v^{2} q^{c d} n_{c} n_{d}\left(\epsilon q^{a b}+p^{a b}\right)-Q^{a b}\right] \iota_{b}
$$

for $\iota_{b}$ a unit polarization vector. Then the weak energy condition, $T_{a b} b^{a} l^{b} \geq 0$ for all null $l^{a}$, implies $\left(\epsilon^{a b}+p^{a b}\right) \xi_{a}{ }^{*} \xi_{b}$ $\geq 0$. Requiring $v^{2}<1$ for all principal sound velocities gives

$$
\mathscr{A}_{E \mathrm{~S}} \geq\left[\left(u^{a} n_{a}\right)^{2}-q^{c d} n_{c} n_{d}\right]\left(\epsilon q^{a b}+p^{a b}\right) \xi_{a}{ }^{*} \xi_{b},
$$

positive-definite when $n^{a}$ is nonspacelike.

\section{APPENDIX III}

We present here a derivation of the formulae in $\S$ IIIl $b$ leading to the symmetry relation (3.18). The following operators defined on tensor fields $Q$ will be useful:

and

$$
\delta Q=(-g)^{-1 / 2} \delta\left[Q(-g)^{1 / 2}\right], \quad £_{n} W=(-g)^{-1 / 2} £_{n}\left[Q(-g)^{1 / 2}\right],
$$

$$
\Delta Q=(-g)^{-1 / 2} \Delta\left[Q(-g)^{1 / 2}\right]=\left(\delta+f_{\xi}\right) Q .
$$

Let us first establish equation (3.12), namely,

We have

$$
\delta G^{a b}=\frac{1}{2} \epsilon^{a c e g} \epsilon^{b d f} \nabla_{(c)} \nabla_{d} h_{e f}+G^{a b c d} h_{c d} .
$$

$$
\begin{aligned}
\delta G^{a b} & =(-g)^{-1 / 2} \delta\left[R_{c d}\left(g^{a c} g^{b d}-\frac{1}{2} g^{a b} g^{c d}\right)(-g)^{1 / 2}\right] \\
& =\delta R_{c a}\left(g^{a c} g^{b d}-\frac{1}{2} g^{a b} g^{c d}\right)+\frac{1}{2} h\left(R^{a b}-\frac{1}{2} g^{a b} R\right)-\left(h^{a c} g^{b d}+g^{a c} h^{b d}-\frac{1}{2} h^{a b} g^{c d}-\frac{1}{2} g^{a b} h^{c d}\right) R_{c d} .
\end{aligned}
$$

By means of the relation (eq. [8.11])

$$
\delta R_{a b}=\nabla^{c} \nabla_{(a} h_{b) c}-\frac{1}{2} \nabla_{a} \nabla_{b} h-\frac{1}{2} \nabla^{c} \nabla_{c} h_{a b}
$$


the first term on the right-hand side of equation (A28) can be written as

$$
\begin{aligned}
\delta R_{c d}\left(g^{a c} g^{b d}-\frac{1}{2} g^{a b} g^{c d}\right) & \\
= & \frac{1}{2}\left(g^{a c} g^{b d} g^{e f}-\frac{1}{2} g^{a b} g^{c d} g^{e f}\right)\left(\nabla_{e} \nabla_{c} h_{d f}+\nabla_{e} \nabla_{d} h_{e f}-\nabla_{c} \nabla_{d} h_{e f}-\nabla_{e} \nabla_{f} h_{c d}\right) \\
= & \frac{1}{2} \nabla_{(c} \nabla_{d)} h_{e f}\left(g^{a b} g^{c d} g^{e f}+g^{a f} g^{c b} g^{e d}+g^{a d} g^{c f} g^{e b}-g^{a b} g^{c f} g^{e d}-g^{a d} g^{c b} g^{e f}-g^{a f} g^{c d} g^{e b}\right) \\
& +\frac{1}{2} \nabla_{[c} \nabla_{d j}\left(g^{a f} g^{b d} g^{c e}+g^{a d} g^{c f} g^{b e}-g^{a b} g^{c f} g^{e d}-g^{a c} g^{b d} g^{e f}\right) \\
= & \frac{1}{2} \epsilon^{a c e g} \epsilon^{b d f} \nabla_{(c} \nabla_{d j} h_{e f}-\frac{1}{2} R^{a c b d} h_{c d}+\frac{1}{2} R^{c(a} h_{c}^{b)} .
\end{aligned}
$$

Substituting this expresion in equation (A29) and grouping like terms, we obtain

where

$$
\begin{aligned}
\delta G^{a b}= & \frac{1}{2} \epsilon^{a c e g} \epsilon^{b d f} \nabla_{(c} \nabla_{d} h_{e f}-\frac{1}{2} R^{a c b d} h_{c d}+\frac{1}{2}\left(g^{a b} R^{c d}+g^{c d} R^{a b}-g^{a c} R^{b d}-g^{b c} R^{a d}\right) h_{c d} \\
& +\frac{1}{4} R\left(g^{a c} g^{b d}+g^{a d} g^{b c}-g^{a b} g^{c d}\right) h_{c d} \\
= & \frac{1}{2} \epsilon^{a c e g} \epsilon^{b d{ }_{g}} \nabla_{(c} \nabla_{d)} h_{e f}+G^{a b c d} h_{c d},
\end{aligned}
$$

$$
\begin{aligned}
G^{a b c d} & =\frac{1}{2} R^{a(c d) b}+\frac{1}{2}\left(g^{a b} R^{c d}+g^{c d} R^{a b}-g^{a(c} R^{d) b}-g^{b(c} R^{d) a}\right)+\frac{1}{4} R\left(g^{a c} g^{b d}+g^{a d} g^{b c}-g^{a b} g^{c d}\right) \\
& =G^{(a b)(c d)}=G^{c d a b} ;
\end{aligned}
$$

as a result, the expression obtained from equation (A31) by contraction with an arbitrary tensor $\bar{h}_{\mathrm{a} b}$ is manifestlv symmetric in $h_{a b}$ and $h_{a b}$ to within a divergence:

$$
\bar{h}_{a b} \delta G^{a b}=-\frac{1}{2} \epsilon^{a c e g} \epsilon^{b d f} \nabla_{c} h_{a b} \nabla_{d} h_{e f}+G^{a b c d} h_{a b} h_{c d}+\nabla_{c}\left(\frac{1}{2} \epsilon^{a c e g} \epsilon^{b d f}{ }_{o} \bar{h}_{a b} \nabla_{d} h_{e f}\right) .
$$

We next derive the analogous relations for the fluid perturbations. The equations of $\S$ III $a$,

and

$$
\Delta u^{a}=\frac{1}{2} u^{a} u^{b} u^{c} \Delta g_{b c}
$$

$$
\frac{\Delta p}{\gamma p}=\frac{\Delta \epsilon}{\epsilon+p}=-\frac{1}{2} q^{a b} \Delta g_{a b},
$$

permit the Lagrangian change in the energy momentum tensor,

$$
T^{\mathrm{ab}}=(\epsilon+p) u^{\mathrm{a}} u^{\mathrm{b}}+p g^{\mathrm{ab}}=\epsilon u^{\mathrm{a}} u^{\mathrm{b}}+p q^{\mathrm{ab}}
$$

to be expressed in terms of the variables $\xi^{a}$ and $h_{a b}$ via the perturbed metric $\Delta g_{a b}=h_{a b}+2 \nabla_{(a} \xi_{b}$. In particular, observing first that

we have

$$
\Delta q^{a b}=\left(-g^{a c} g^{b d}+u^{a} u^{b} u^{c} u^{d}\right) \Delta g_{c d}
$$

$$
\begin{aligned}
\Delta T^{a b} & =\Delta \epsilon u^{a} u^{b}+2 \epsilon u^{(a)} \Delta u^{b)}+\Delta p q^{a b}+p \Delta q^{a b} \\
& =\left[-\frac{1}{2}(\epsilon+p) q^{c d} u^{a} u^{b}+\epsilon u^{a} u^{b} u^{c} u^{d}-\gamma p q^{c d} q^{a b}+p\left(-g^{a c} g^{b d}+u^{a} u^{b} u^{c} u^{d}\right)\right] \Delta g_{c d} .
\end{aligned}
$$

Now

Consequently,

$$
(-g)^{-1 / 2} \Delta(-g)^{1 / 2}=\frac{1}{2} g^{a b} \Delta g_{a b} .
$$

$$
\begin{aligned}
\Delta T^{a b} & =\Delta T^{a b}+\frac{1}{2} T^{a b} g^{c d} \Delta g_{c d} \\
& =\left[\frac{1}{2}(\epsilon+p) u^{a} u^{b} u^{c} u^{d}+\frac{1}{2} p\left(g^{a b} g^{c d}-g^{a c} g^{b d}-g^{a d} g^{b c}\right)-\frac{1}{2} \gamma p q^{a b} q^{c d}\right] \Delta g_{c d} \\
& \equiv W^{a b c d} \Delta g_{c d},
\end{aligned}
$$

where

$$
W^{a b c d}=W^{(a b)(c d)}=W^{c d a b} .
$$

An analog of the relation (A33), namely,

then follows.

$$
\bar{\Delta} g_{a b} \Delta T^{a b}=W^{a b c d} \bar{\Delta} g_{a b} \Delta g_{c d}=\Delta g_{a b} \bar{\Delta} T^{a b},
$$


Equation (A39) is equivalent to a relation of the form

$\bar{\xi}_{a} \Delta \nabla_{b} T^{a b}-\frac{1}{2} \bar{h}_{a b} \delta T^{a b}=$ expression symmetric in the barred and unbarred quantities $\}+$ divergence .

In showing the equivalence we require the following lemma:

$$
\bar{\xi}_{a}\left[\Delta, \nabla_{b}\right] T^{a b}=-T^{a b}\left[\nabla_{a} \bar{\xi}_{c} \nabla_{b} \xi^{c}-R_{a c b d} \bar{\xi}^{c} \xi^{d}\right]+\frac{1}{2} \xi_{\xi} T^{\overline{a b}}+\nabla_{a}\left[T^{a b} \bar{\xi}^{c} h_{b c}+T^{a b} \bar{\xi}^{c} \nabla_{b} \xi_{c}-\frac{1}{2} T^{b c} \bar{\xi} a h_{b c}\right] .
$$

To prove the lemma, we note first that the commutation relation

$$
\left[£_{\xi}, \nabla_{b}\right] \eta^{a}=R_{b c d}^{a} \eta^{c} \xi^{d}+\eta^{c} \nabla_{c} \nabla_{b} \xi^{a},
$$

together with the fact that $\left[f_{\xi}, \nabla_{b}\right]$ is a derivation implies the identity

$$
\left[\Delta, \nabla_{b}\right] A^{a_{1} \cdots a_{n}}=\sum_{i=1}^{n}\left[\delta \Gamma_{b c}^{a d}+\nabla_{c} \nabla_{b} \xi^{a t}+R_{b d c}^{a i} \xi^{d}\right] A^{a_{1} \cdots a_{t-1} c a_{i+1} \cdots a_{n}}
$$

where

Hence

$$
\delta \Gamma_{b c}^{a} \equiv \frac{1}{2} g^{a d}\left(\nabla_{c} h_{b d}+\nabla_{b} h_{c d}-\nabla_{d} h_{b c}\right) \text {. }
$$

$$
\left[\Delta, \nabla_{b}\right] T^{a b}=\left[\frac{1}{2}\left(\nabla_{c} h_{b}^{a}+\nabla_{b} h_{c}^{a}-\nabla^{a} h_{b c}\right)+\nabla_{c} \nabla_{b} \xi^{a}-R_{b c d}^{a} \xi^{d}\right] T^{b c}+\nabla_{b}\left(\frac{1}{2} h+\nabla_{c} \xi^{c}\right) T^{a b}
$$

But

whence

$$
(-g)^{-1 / 2}\left[\Delta, \nabla_{b}\right](-g)^{1 / 2}=-\nabla_{b}\left(\frac{1}{2} h+\nabla_{c} \xi^{c}\right),
$$

Thus

$$
\left[\Delta, \nabla_{b}\right] T^{a b}=T^{b c}\left[\frac{1}{2}\left(\nabla_{c} h^{a}{ }_{b}+\nabla_{b} h^{a}{ }_{c}-\nabla^{a} h_{b c}\right)+\nabla_{c} \nabla_{b} \xi^{a}-R^{a}{ }_{b c d} \xi^{d}\right] .
$$

$$
\bar{\xi}_{a}\left[\Delta, \nabla_{b}\right] T^{a b}=T^{a b}\left[\bar{\xi}^{c} \nabla_{\alpha} \nabla_{b} \xi_{c}+R_{a c b d} \bar{\xi}^{c} \xi^{d}\right]+\frac{1}{2}\left(\nabla_{c} h_{a b}+\nabla_{b} h_{a c}-\nabla_{a} h_{b c}\right) \bar{\xi}^{a} T^{b c},
$$

which is equivalent to the lemma when the equilibrium equation $\nabla_{a} T^{a b}=0$ is satisfied.

To obtain equation (A40), we write the relation (A39) in the form

Then

$$
W^{a b c d} \bar{\Delta} g_{a b} \Delta g_{c a}=\left(\bar{h}_{a b}+2 \bar{\xi}_{b} \nabla_{a}\right) \Delta T^{a b}+2 \nabla_{a}\left(\bar{\xi}_{b} \Delta T^{a b}\right) \text {. }
$$

$$
\bar{\xi}_{b} \nabla_{a} \Delta T^{a b}-\frac{1}{2} h_{a b} \bar{\delta} T^{a b}=-\frac{1}{2} W^{a b c d} \bar{\Delta} g_{a b} \Delta g_{c d}+\frac{1}{2} \bar{h}_{a b} \boldsymbol{f}_{\xi} T^{a b}+\nabla_{a}\left(\bar{\xi}_{b} \Delta T^{a b}\right),
$$

and the lemma implies that

$$
\begin{aligned}
\bar{\xi}_{b} \Delta \nabla_{a} T^{a b}-\frac{1}{2} \bar{h}_{a b} \delta T^{a b} & \\
= & -T^{a b}\left(\nabla_{a} \bar{\xi}^{c} \nabla_{b} \xi_{c}-R_{a c b d} \bar{\xi}^{c} \xi^{d}\right)+\frac{1}{2}\left(h_{a b} \mathfrak{f}_{\xi} T^{a b}+h_{a b} \boldsymbol{f}_{\bar{\xi}} T^{a b}\right)-\frac{1}{2} W^{a b c d} \bar{\Delta}_{a b} \Delta g_{c d} \\
& +\nabla_{a}\left[T^{a b} \xi^{c}\left(h_{b c}+\nabla_{b} \xi_{c}\right)-\frac{1}{2} T^{o c} \bar{\xi}^{a} h_{b c}+W^{a b c d} \xi_{b}\left(h_{c d}+\nabla_{c} \xi_{d}\right)\right] .
\end{aligned}
$$

Making use of equation (A44) and of the relation

we obtain

$$
\mathfrak{f}_{\xi} T^{a b}=\xi^{c} \nabla_{c} T^{a b}-\nabla_{c} \xi^{a} T^{b c}-\nabla_{c} \xi^{b} T^{a c}+\nabla_{c} \xi^{c} T^{a b},
$$

$$
\begin{aligned}
\bar{\xi}_{b} \Delta \nabla_{b} T^{a b}-\frac{1}{2} h_{a b} \delta T^{a b} & \\
= & -U^{a b c d} \nabla_{a} \bar{\xi}_{b} \nabla_{c} \bar{\xi}_{d}-V^{a b c d}\left(h_{a b} \nabla_{c} \xi_{d}+h_{a b} \nabla_{c} \bar{\xi}_{a}\right)-\frac{1}{2} W^{a b c d} h_{a b} h_{c d}+T^{a b} R_{a c b d} \bar{\xi}^{c} \xi^{d} \\
& +\frac{1}{2} \nabla_{c} T^{a b}\left(\bar{\xi}^{c} h_{a b}+\xi^{c} \bar{h}_{a b}\right)+\nabla_{a}\left(U^{a b c d} \bar{\xi}_{b} \nabla_{c} \xi_{d}+V^{c d a b} h_{c d} \bar{\xi}_{b}\right)
\end{aligned}
$$

where

$$
\begin{aligned}
U^{a b c d} & =2 W^{a b c d}+T^{a c} g^{b d} \\
& =(\epsilon+p) u^{a} u^{c} q^{b d}+p\left(g^{a b} g^{c d}-g^{a d} g^{b c}\right)-\gamma p q^{a b} q^{c d}=U^{c d a b}
\end{aligned}
$$

and

$$
\begin{aligned}
V^{a b c d} & =W^{a b c d}+\frac{1}{2}\left(T^{a c} g^{b d}+T^{b c} g^{a d}-T^{a b} g^{c d}\right) \\
& \left.=\frac{1}{2}(\epsilon+p) u^{a} u^{c} q^{b d}+u^{b} u^{c} q^{a d}-u^{a} u^{b} q^{c d}\right)-\frac{1}{2} \gamma p q^{a b} q^{c d}
\end{aligned}
$$


Finally, multiplying equation (A51) by $16 \pi$ and adding it to equation (A33), we obtain the first relation of $\S \mathrm{II} b$, equation (3.18):

$$
\begin{aligned}
16 \pi \bar{\xi}_{b} \Delta \nabla_{a} T^{a b} & +\bar{h}_{a b} \delta\left(G^{a b}-8 \pi T^{a b}\right) \\
= & -16 \pi U^{a b c d} \nabla_{a} \bar{\xi}_{b} \nabla_{c} \xi_{d}-16 \pi V^{a b c d}\left(\bar{h}_{a b} \nabla_{c} \xi_{d}+h_{a b} \nabla_{c} \bar{\xi}_{d}\right)-\frac{1}{2} \epsilon^{a c e g} \epsilon^{b d f} \nabla_{c} \bar{h}_{a b} \nabla_{d} h_{e f} \\
& +8 \pi \nabla_{c} T^{a b}\left(\bar{h}_{a b} \xi^{c}+h_{a b} \bar{\xi}^{c}\right)+16 \pi T^{a b} R_{a c b d} \bar{\xi}^{c} \xi^{d}+\left(G^{a b c d}-8 \pi W^{a b c d}\right) \bar{h}_{a b} h_{c d} \\
& +\nabla_{a}\left[16 \pi U^{a b c d} \bar{\xi}_{b} \nabla_{c} \xi_{d}+16 \pi V^{c d a b} h_{c d} \bar{\xi}_{b}+\frac{1}{2} \epsilon^{a c e g} \epsilon^{b d f}{ }_{g} \bar{h}_{c d} \nabla_{b} h_{e f}\right] \\
= & 16 \pi \bar{\xi}_{b} \Delta \nabla_{a} T^{a b}+\bar{h}_{a b} \delta\left(G^{a b}-8 \pi T^{a b}\right),
\end{aligned}
$$

where in the last line the equilibrium field equations have been used to regain the ordinary Lagrangian and Eulerian operators.

\section{REFERENCES}

Araki, H. 1959, Ann. Phys., 7, 456.

Bardeen, J. M., and Wagoner, R. V. 1971, Ap. J., 167, 359.

Brecher, K., and Morrison, P. 1973, Ap. J., 180, L107.

Carter, B. 1973a, Commun. Math. Phys., 30, 261.

- 1973b, Phys. Rev. D., 7, 1590.

Carter, B., and Quintana, H. 1972, Prov. Roy. Soc. (London) A, 331, 57.

Chandrasekhar, S. 1961, Hydrodynamic and Hydromagnetic Stability (Oxford: Clarendon Press). 1964, Ap. J., 139, 664.

1969, Ellipsoidal Figures of Equilibrium (New Haven, Conn.: Yale University Press).

. 1970, Phys. Rev. Letters, 24, 611.

Chandrasekhar, S., and Elbert, D. D. 1974 Ap. J., 192, 731.

Chandrasekhar, S., and Friedman, J. L. 1972a, Ap. J., 175, 379.

$1972 b$, Ap. J., $176,745$.

Chandrasekhar, S., and Lebovitz, N. R. 1964, Ap. J., 140, 1517.

1973, ibid., $185,19$.

Clement, M. 1964, Ap. J., 140, 1045.

1965, ibid., 141, 210.

Detweiler, S., and Ipser, J. R. 1973, Ap. J., 185, 685.

Friedman, J. L. 1975 (to be published).

Friedman, J. L., and Schutz, B. 1975 (to be published).

Geroch, R. 1972, J. Math. Phys. 13, 956.

Hartle, J. B., and Wilkins, D. C. 1974, Comm. Math. Phys. $38,47$.

Ipser, J. R. 1975, $A$ p. J., in press.

Ipser, J. R., and Butterworth, M. 1975 (to be published).

Kulsrud, R. M. 1968, Ap.J., 152, 1121.

Lebovitz, N. R. 1961, Ap. J., 134, 500.

Lynden-Bell, D., and Ostriker, J. P. 1967, M.N.R.A.S., 136, 293.

Newman, E. T., and Unti, T. W. J. 1962, J. Math. Phys

891.

Sachs, R. K. 1962, Proc. Roy. Soc. (London) A, 270, 103.

Schutz, B. F. 1970, Phys. Rev. D., 2, 2762.

Taub. 1972, Ap. J. Suppl., No. 208, 24, 343.

Taub, A. H. 1954, Phys. Rev., 94, 1468.

. 1969, Commun. Math. Phys., 15, 235.

Wheeler, J. C., and Rosenwald, R. D. 1973, Ap. Letters, 15, 75.

Whittaker, E. T. 1937, A Treatise on the Analytical Dynamics of Particles and Rigid Bodies (Cambridge: Cambridge University Press)

Will, C. M. 1973, Ap. J., 190, 403.

Wilson, J. R. 1972, Ap.J., 176, 195

John L. Friedman: Laboratory for Astrophysics and Space Research, 933 E. 56th Street, Chicago, IL 60637

Bernard F. SChutz: Department of Applied Mathematics and Astronomy, University College, P.O. Box 78, Cardiff, CF1 1XL, Great Britain 


\section{ERRATA}

In the paper "The Characteristic Size of Clusters of Galaxies: A Metric Rod Used for a Determination of $q_{0}$ " by Gustavo Bruzual A. and Hyron Spinrad (Ap.J., 220, 1 [1978]), an inconsistent application of values of 50 and $100 \mathrm{~km} \mathrm{~s}^{-1} \mathrm{Mpc}^{-1}$ for the Hubble constant was inadvertently made.

Equations (7) and (8) should read:

and

$$
\begin{aligned}
q_{0} & =0.23 \pm 0.51, \\
l_{c} & =(446 \pm 12) h_{50}^{-1} \mathrm{kpc}, \\
q_{0} & =0.27 \pm 0.58, \\
l_{c} & =(293 \pm 25) h_{50}^{-1} \mathrm{kpc},
\end{aligned}
$$

respectively.

The values of $l_{c}$ in Table 2 (col. [10]) correspond to $H_{0}=55 \mathrm{~km} \mathrm{~s}^{-1} \mathrm{Mpc}^{-1}\left(h_{0}=1.1\right)$, and the legend to Figure 4 should read: "Angular size plotted as a function of redshift for data given in columns (4), (7), and (8) of Table 1. Curves given by eqs. (2) for $l_{c}=446 h_{50}^{-1} \mathrm{kpc}, h_{50}=1.1$, and $q_{0}=0,0.50$, and 1 are shown. The error bars correspond to $\pm \sigma(\log a)$. The available data suggest $q_{0}{ }^{\mathrm{appr}}=+0.25$."

We thank Dr. P. Henry for pointing out the factor of 2 inconsistency in equations (7) and (8) and the text.

The paper, "On the Stability of Relativistic Systems" by John L. Friedman and Bernard F. Schutz (Ap. J., 200, 204 [1975]) contained several sign and arithmetic errors which do not affect the paper's conclusions. The following is what the authors believe to be a complete catalog of them.

1. In the last line on p. 205 , change $R^{i}$ to $-R^{t}$.

2. The definition of $G_{a b c d}$ following equation (3.12) should read

$$
\begin{aligned}
G_{a b c d}= & \frac{1}{2} R_{a(c d) b}-\frac{1}{2}\left[\frac{3}{2} R_{a(c} g_{d) b}+\frac{3}{2} R_{b(c c} g_{d)} a-R_{a b} g_{c d}-R_{c d} g_{a b}\right] \\
& +\frac{1}{4} R\left(g_{a c} g_{b d}+g_{a d} g_{b c}-g_{a b} g_{c d}\right) .
\end{aligned}
$$

Note that $G^{a b c}{ }_{c}=0$.

3. Everywhere the product $\epsilon^{\text {aceg }} \epsilon^{\text {bdf }}{ }_{g}$ appears on pages $209,210,211,217,218$, and 220, its sign should be changed. The $\epsilon$-terms in Appendix I remain unchanged.

4. In the definition of $\mathscr{C}$ following equation (3.19), $R_{a b c d}$ should be $R_{a c b d}$.

5. In the equation at the bottom of p. $213,10^{-7}$ should be replaced by $10^{-3}$.

6. In the last sentence on p. 217 , the reference to equation (8.11) should be to (3.11).

7. In the second-to-last line of equation (A30), insert $h_{e f}$ immediately following $\nabla_{c c} \nabla_{d]}$.

8. In the sentence following equation (A30) the reference to (A29) should be (A28); in the equation on the next line insert $3 / 2$ in front of $g^{a c} R^{b d}$ and $g^{b c} R^{a d}$.

9. In equation (A32), the definition of $G^{a b c d}$, insert $3 / 2$ in front of $g^{a(c} R^{d) b}$ and $g^{b(c} R^{d) a}$.

10. In the equation between (A41) and (A42) the sign of $R^{a}{ }_{b c d}$ is wrong.

11. In equation (A47) change the first + to - .

12. In equation (A48) $\bar{\delta} T^{a b}$ should be $\delta T^{a b}$.

13. In equation (A49) the final term should be $W^{a b c d} \bar{\xi}_{b}\left(h_{c d}+2 \nabla_{c} \xi_{d}\right)$.

14. In the final line of equation (A52), insert a left parenthesis immediately after $(\epsilon+p)$. 